\title{
BISIMULATIONS MEET PCTL EQUIVALENCES FOR PROBABILISTIC AUTOMATA*
}

\author{
LEI SONG ${ }^{a}$, LIJUN ZHANG $^{b}$, JENS CHR. GODSKESEN $^{c}$, AND FLEMMING NIELSON $^{d}$ \\ ${ }^{a}$ Max-Planck-Institut für Informatik, and Saarland University - Computer Science, Germany \\ e-mail address: song@cs.uni-saarland.de \\ ${ }^{b}$ State Key Laboratory of Computer Science, Institute of Software, Chinese Academy of Sciences, \\ DTU Informatics, Technical University of Denmark, and Saarland University - Computer Science, \\ Germany \\ e-mail address: zhang@imm.dtu.dk and zhanglj@ios.ac.cn (corresponding author) \\ ${ }^{c}$ IT University of Copenhagen, Denmark \\ e-mail address: jcg@itu.dk \\ ${ }^{d}$ DTU Compute, Technical University of Denmark \\ e-mail address: fnie@dtu.dk
}

\begin{abstract}
Probabilistic automata (PAs) have been successfully applied in formal verification of concurrent and stochastic systems. Efficient model checking algorithms have been studied, where the most often used logics for expressing properties are based on probabilistic computation tree logic (PCTL) and its extension PCTL*. Various behavioral equivalences are proposed, as a powerful tool for abstraction and compositional minimization for PAs. Unfortunately, the equivalences are well-known to be sound, but not complete with respect to the logical equivalences induced by PCTL or PCTL*. The desire of a both sound and complete behavioral equivalence has been pointed out by Segala in 34, but remains open throughout the years. In this paper we introduce novel notions of strong bisimulation relations, which characterize PCTL and PCTL* exactly. We extend weak bisimulations that characterize PCTL and PCTL* without next operator, respectively. Further, we also extend the framework to simulation preorders. Thus, our paper bridges the gap between logical and behavioral equivalences and preorders in this setting.
\end{abstract}

2012 ACM CCS: [Mathematics of computing]: Probability and statistics-Stochastic processesMarkov processes; [Theory of computation]: Logic-Modal and temporal logics; Semantics and reasoning-Program reasoning-Program verification; [General and reference]: Cross-computing tools and techniques-Performance; Cross-computing tools and techniques-Verification.

Key words and phrases: PCTL, Probabilistic automata, Characterization, Bisimulation.

* An extended abstract of the paper has appeared in [37. 


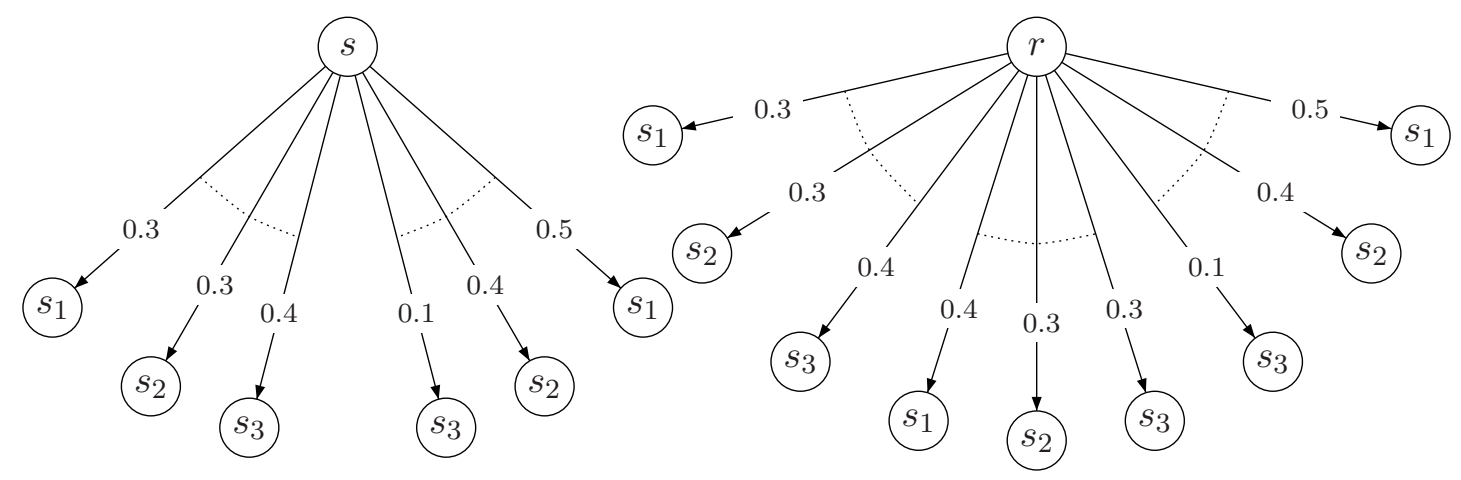

Figure 1: Counterexample of strong probabilistic bisimulation.

\section{INTRODUCTION}

Probabilistic automata (PAs) 35 have been successfully applied in formal verification of concurrent and stochastic systems. Efficient model checking algorithms have been studied, where properties are mostly expressed by the probabilistic computation tree logic (PCTL) [16] and its extension PCTL* [1] for Markov chains, and later extended in [6] for Markov decision processes.

To combat the infamous state space problem in model checking, various behavioral equivalences, including strong and weak bisimulations, are proposed for stochastic models including PAs [26, 25, 34, 3, 35]. Indeed, they turn out to be a powerful tool for abstraction for PAs, since bisimilar states imply that they satisfy exactly the same PCTL and PCTL* formulas, thus can be grouped together, allowing one to construct smaller quotient automata before analyzing the models. In practice, bisimulation based minimization is extensively studied in the literatures to leverage the state space explosion, for instance see [8, 2, 24]. Moreover, the nice compositional theory for PAs is exploited for compositional minimization [7], namely minimizing the automata before composing the components together.

An interesting question is whether the reverse holds as well, namely whether logical equivalences imply bisimulation equivalences? For Markov chains, i.e., PAs without nondeterministic choices, the answer is affirmative [1, 5]. Unfortunately, the completeness does not hold in general, namely PCTL equivalence is strictly coarser than bisimulation and its variant probabilistic bisimulation [35] for PAs.

The main reason for the gap can be illustrated by the following example. Consider the PA in Fig. 1 assuming that $s_{1}, s_{2}, s_{3}$ are three absorbing states with different state properties. It is easy to see that $s$ and $r$ are PCTL equivalent: the additional middle transition out of $r$ does not change the extreme probabilities, the intervals of probabilities in which the three observing states can be reached are not changed. However existing bisimulations differentiate $s$ and $r$, mainly because the middle transition out of $r$ cannot be matched by any transition (or combined transition) of $s$. Bisimulation requires that the complete distribution of a transition must be matched, which is in this case too strong, as it differentiates states satisfying the same PCTL formulas.

For PAs, the desire of a both sound and complete behavioral equivalence has been pointed out in [34] (see section 13.2.7), but remains open throughout the years. Such a sound and complete relation is not only of theoretical interests: in practice it would allow us to construct the minimal quotient automata for checking PCTL and PCTL* formulas, which are arguably the most often used logics for specifying properties over PAs. In this 
paper we bridge this gap by introducing novel notions of behavioral equivalences which characterize (both soundly and completely) PCTL, PCTL* and their sub logics. Summarizing, our contributions are:

- A new bisimulation characterizing PCTL* soundly and completely. The bisimulation arises from a converging sequence of equivalence relations, each of which characterizes bounded PCTL*.

- Branching bisimulations which correspond to PCTL and bounded PCTL equivalences.

- We then extend our definitions to weak bisimulations, which characterize sub logics of PCTL and PCTL* with only unbounded path formulas.

- Further, we extend the framework to simulations as well as their characterizations, extend the results to countable states, and discuss the coarsest congruent bisimulation and simulation relations.

Organization of the paper. Section 2 introduces some notations. In Section 3 we recall definitions of probabilistic automata and bisimulation relations by Segala [34. We also recall the logic PCTL* and its sub logics. Section 4 introduces the novel strong and strong branching bisimulations, and proves that they agree with PCTL* and PCTL equivalences, respectively. Section 5 extends them to weak (branching) bisimulations, and Section 6 extends the framework to simulations. We discuss the extension to countable states in Section 7 and the coarsest congruent bisimulations and simulations in Section 8 . In Section 9 we discuss related work, and Section 10 concludes the paper.

\section{Preliminaries}

Distributions. For a countable set $S$, a distribution is a function $\mu: S \rightarrow[0,1]$ satisfying $|\mu|:=\sum_{s \in S} \mu(s)=1$. We denote by $\operatorname{Dist}(S)$ the set of distributions over $S$. We shall use $s, r, t, \ldots$ and $\mu, \nu \ldots$ to range over $S$ and $\operatorname{Dist}(S)$, respectively. Given a set of distributions $\left\{\mu_{i}\right\}_{1 \leq i \leq n}$, and a set of positive weights $\left\{w_{i}\right\}_{1 \leq i \leq n}$ such that $\sum_{1<i<n} w_{i}=1$, the convex combination $\mu=\sum_{1 \leq i \leq n} w_{i} \cdot \mu_{i}$ is a distribution such that $\mu(s)=\sum_{1 \leq i \leq n} w_{i} \cdot \mu_{i}(s)$ for each $s \in S$. The support of $\mu$ is defined by $\operatorname{supp}(\mu):=\{s \in S \mid \mu(s)>0\}$. For an equivalence relation $\mathcal{R}$, we write $\mu \mathcal{R} \nu$ if it holds that $\mu(C)=\nu(C)$ for all equivalence classes $C \in S / \mathcal{R}$. A distribution $\mu$ is called Dirac if $|\operatorname{supp}(\mu)|=1$, and we let $\mathcal{D}_{s}$ denote the Dirac distribution with $\mathcal{D}_{s}(s)=1$.

Downward Closure. We define the downward closure of a set of states. For a relation $\mathcal{R}$ over $S$ and $C \subseteq S$, define $\mathcal{R}^{\downarrow}(C)$ as the least set satisfying: i) $C \subseteq \mathcal{R}^{\downarrow}(C)$, ii) $\left(s, s^{\prime}\right) \in \mathcal{R}$ and $s^{\prime} \in \mathcal{R}^{\downarrow}(C)$ implies $s \in \mathcal{R}^{\downarrow}(C)$. We say $C$ is $\mathcal{R}$ downward closed iff $C=\mathcal{R}^{\downarrow}(C)$. We use $\mathcal{R}^{\downarrow}(s)$ as the shorthand of $\mathcal{R}^{\downarrow}(\{s\})$, and $\mathcal{R}^{\downarrow}=\left\{C \mid C \subseteq S \wedge C=\mathcal{R}^{\downarrow}(C)\right\}$ to denote the set of all $\mathcal{R}$ downward closed sets. If $\mathcal{R}$ is an equivalence relation, then $C$ is called $\mathcal{R}$ closed if $C=\mathcal{R}^{\downarrow}(C)$. 


\section{Probabilistic automata, PCTL* and Bisimulations}

3.1. Probabilistic automata. We recall the notion of probabilistic automata introduced by Segala 34. We omit the set of actions, since they do not appear in the logic PCTL we shall consider later. This is actually not a restriction, since the bisimulation we shall introduce later can be extended to PAs with actions directly.

Definition 3.1. A probabilistic automaton is a tuple $\mathcal{P}=\left(S, \rightarrow, s_{0}, A P, L\right)$ where $S$ is a finite set of states, $\rightarrow \subseteq S \times \operatorname{Dist}(S)$ is a transition relation such that for each $s \in S$, there exists $(s, \mu) \in \rightarrow$ for some $\mu, s_{0} \in S$ is the initial state, $A P$ is a set of atomic propositions, and $L: S \rightarrow 2^{A P}$ is a labeling function.

We only consider image-finite PAs, i.e. $\{\mu \mid(s, \mu) \in \rightarrow\}$ is finite for each $s \in S$. A transition $(s, \mu) \in \rightarrow$ is often denoted by $s \rightarrow \mu$. Moreover, we write $\mu \rightarrow \mu^{\prime}$ iff for each $s \in \operatorname{supp}(\mu)$ there exists $s \rightarrow \mu_{s}$ such that $\mu^{\prime}(r)=\sum_{s \in \operatorname{supp}(\mu)} \mu(s) \cdot \mu_{s}(r)$.

A path is a finite or infinite sequence $\omega=s_{0} s_{1} s_{2} \ldots$ of states. We use lstate $(\omega)$ and $l(\omega)$ to denote the last state of $\omega$ and the length of $\omega$ respectively if $\omega$ is finite. The set Path is the set containing all paths, and Path $\left(s_{0}\right)$ contains those starting from $s_{0}$. Similarly, Path* is the set of finite paths, and $\operatorname{Path}^{*}\left(s_{0}\right)$ only contains finite paths starting from $s_{0}$. Also we use $\omega[i]$ to denote the $(i+1)$-th state for $i \geq 0,\left.\omega\right|^{i}$ to denote the prefix of $\omega$ ending at $\omega[i]$, and $\left.\omega\right|_{i}$ to denote the suffix of $\omega$ starting from $\omega[i]$.

We introduce the definition of schedulers to resolve non-determinism. A scheduler is a function $\sigma:$ Path $^{*} \mapsto \operatorname{Dist}(\rightarrow)$ such that $\sigma(\omega)(s, \mu)>0$ implies $s=$ lstate $(\omega)$. A scheduler $\sigma$ is deterministic if it returns only Dirac distributions, that is, the next step is chosen deterministically.

The cone of a finite path $\omega$, denoted by $C_{\omega}$, is the set of paths having $\omega$ as their prefix, i.e., $C_{\omega}=\left\{\omega^{\prime} \mid \omega \leq \omega^{\prime}\right\}$ where $\omega \leq \omega^{\prime}$ iff $\omega$ is a prefix of $\omega^{\prime}$. Fixing a starting state $s_{0}$ and a scheduler $\sigma$, the measure $\operatorname{Prob}_{\sigma, s_{0}}$ of a cone $C_{\omega}$, where $\omega=s_{0} s_{1} \ldots s_{k}$, is defined inductively as follows: $\operatorname{Prob}_{\sigma, s_{0}}\left(C_{\omega}\right)$ equals 1 if $k=0$, and for $k>0$,

$$
\operatorname{Prob}_{\sigma, s_{0}}\left(C_{\omega}\right)=\operatorname{Prob}_{\sigma, s_{0}}\left(C_{\left.\omega\right|^{k-1}}\right) \cdot\left(\sum_{\left(s_{k-1}, \mu^{\prime}\right) \in \rightarrow} \sigma\left(\left.\omega\right|^{k-1}\right)\left(s_{k-1}, \mu^{\prime}\right) \cdot \mu^{\prime}\left(s_{k}\right)\right)
$$

Let $\mathcal{B}$ be the smallest algebra that contains all the cones and is closed under complement and countable unions. By standard measure theory [14, 31] this algebra is a $\sigma$-algebra and all its elements are measurable sets of paths. Given a scheduler $\sigma, \operatorname{Prob}_{\sigma, s_{0}}$ can be extended to a unique measure on $\mathcal{B}$.

Given a relation $\mathcal{R}$ over $S,\left(\mathcal{R}^{\downarrow}\right)^{i}$ is the Cartesian product of $\mathcal{R}^{\downarrow}$ with itself $i$ times. Each element of $\left(\mathcal{R}^{\downarrow}\right)^{i}$ is a downward closed path of length $i$. Let $\left(\mathcal{R}^{\downarrow}\right)^{+}=\cup_{i \geq 1}\left(\mathcal{R}^{\downarrow}\right)^{i}$, and define $l(\Omega)=n$ for $\Omega \in\left(\mathcal{R}^{\downarrow}\right)^{n}$. For $\Omega=C_{0} C_{1} \ldots C_{n} \in\left(\mathcal{R}^{\downarrow}\right)^{+}$, the $\mathcal{R}$ downward closed cone $C_{\Omega}$ is defined as $C_{\Omega}=\left\{C_{\omega} \mid \omega \in \Omega\right\}$, where $\omega \in \Omega$ iff $\omega[i] \in C_{i}$ for $0 \leq i \leq n$.

For distributions $\mu_{1}$ and $\mu_{2}$, we define $\mu_{1} \times \mu_{2}$ by $\left(\mu_{1} \times \mu_{2}\right)\left(\left(s_{1}, s_{2}\right)\right)=\mu_{1}\left(s_{1}\right) \times \mu_{2}\left(s_{2}\right)$. Following [4] we also define the interleaving of PAs:

Definition 3.2. Let $\mathcal{P}_{i}=\left(S_{i}, \rightarrow_{i}, s_{i}, A P_{i}, L_{i}\right)$ be two PAs with $i=1,2$. The interleaved parallel composition $\mathcal{P}_{1} \| \mathcal{P}_{2}$ is defined by:

$$
\mathcal{P}_{1} \| \mathcal{P}_{2}=\left(S_{1} \times S_{2}, \rightarrow,\left(s_{1}, s_{2}\right), A P_{1} \times A P_{2}, L\right)
$$


where $L\left(\left(r_{1}, r_{2}\right)\right)=L_{1}\left(r_{1}\right) \times L_{2}\left(r_{2}\right)$ and $\left(r_{1}, r_{2}\right) \rightarrow \mu$ iff either $r_{1} \rightarrow \mu_{1}$ and $\mu=\mu_{1} \times \mathcal{D}_{r_{2}}$, or $r_{2} \rightarrow \mu_{2}$ and $\mu=\mathcal{D}_{r_{1}} \times \mu_{2}$.

3.2. $\mathrm{PCTL}^{*}$ and its sub logics. We introduce the syntax of PCTL [16] and PCTL* [1] which are probabilistic extensions of CTL and CTL* respectively.

The PCTL* formulas over the set $A P$ of atomic propositions are formed according to the following grammar:

$$
\begin{aligned}
& \varphi::=a\left|\varphi_{1} \wedge \varphi_{2}\right| \neg \varphi \mid \mathcal{P}_{\bowtie q}(\psi) \\
& \psi::=\varphi\left|\psi_{1} \wedge \psi_{2}\right| \neg \psi|\mathrm{X} \psi| \psi_{1} \cup \psi_{2}
\end{aligned}
$$

where $a \in A P, \bowtie \in\{<\rangle,, \leq, \geq\}$, and $q \in[0,1]$. We refer to $\varphi$ and $\psi$ as (PCTL*) state and path formulas, respectively.

The satisfaction relation $s \models \varphi$ for state formulas is defined in a standard manner for boolean formulas. For the probabilistic operator, it is defined by

$$
s \models \mathcal{P}_{\bowtie q}(\psi) \text { iff } \forall \sigma . \operatorname{Prob}_{\sigma, s}(\{\omega \in \operatorname{Path}(s) \mid \omega \models \psi\}) \bowtie q .
$$

The satisfaction relation $\omega \models \psi$ for path formulas is the same as for LTL formulas, that is,

$$
\begin{array}{ll}
\omega \models \mathbf{X} \psi & \text { iff }\left.\omega\right|_{1} \models \psi \\
\omega \models \psi_{1} \cup \psi_{2} & \text { iff } \exists j \geq 0 .\left.\omega\right|_{j} \models \psi_{2} \wedge \forall 0 \leq k<j .\left.\omega\right|_{k} \models \psi_{1}
\end{array}
$$

Sub logics. The depth of path formula $\psi$ of PCTL* free of $U$ operator, denoted by Depth $(\psi)$, is defined by the maximum number of embedded $\mathbf{X}$ operators appearing in $\psi$, that is,

- $\operatorname{Depth}(\varphi)=0$,

- Depth $\left(\psi_{1} \wedge \psi_{2}\right)=\max \left\{\operatorname{Depth}\left(\psi_{1}\right), \operatorname{Depth}\left(\psi_{2}\right)\right\}$,

- Depth $(\neg \psi)=\operatorname{Depth}(\psi)$ and

- $\operatorname{Depth}(\mathbf{X} \psi)=1+\operatorname{Depth}(\psi)$.

Then, we let $\mathrm{PCTL}^{*-}$ be the sub logic of $\mathrm{PCTL}^{*}$ without the until $\left(\psi_{1} \cup \psi_{2}\right)$ operator. Moreover, $\mathrm{PCTL}_{i}^{*-}$ is a sub logic of $\mathrm{PCTL}^{*-}$ where for each $\psi$ we have $\operatorname{Depth}(\psi) \leq i$.

The sub logic PCTL is obtained by restricting the path formulas to:

$$
\psi::=\mathrm{X} \varphi\left|\varphi_{1} \mathrm{U} \varphi_{2}\right| \varphi_{1} \mathrm{U}^{\leq n} \varphi_{2}
$$

Note the bounded until operator does not appear in PCTL* as it can be encoded by nested next operators. $\mathrm{PCTL}^{-}$is defined in a similar way as $\mathrm{PCTL}^{*-}$. Moreover we let $\mathrm{PCTL}_{i}^{-}$be the sub logic of $\mathrm{PCTL}^{-}$where only bounded until operator $\varphi_{1} \mathrm{U}^{\leq j} \varphi_{2}$ with $j \leq i$ is allowed. For all the logics we have mentioned, we summarize their differences in syntax of path formulas in Table 1 .

Logical equivalence. For a logic $\mathcal{L}$, we say that $s$ and $r$ are $\mathcal{L}$-equivalent, denoted by $s \sim_{\mathcal{L}} r$, if they satisfy the same set of formulas of $\mathcal{L}$, that is $s \models \varphi$ iff $r \models \varphi$ for all state formulas $\varphi$ in $\mathcal{L}$. The logic $\mathcal{L}$ can be PCTL* or one of its sub logics. 
Table 1: Summary of PCTL* and its sublogics

\begin{tabular}{|l|c|c|}
\hline \multicolumn{1}{|c|}{ Logic } & $\psi$ & Note \\
\hline PCTL $^{*}$ & $\varphi\left|\psi_{1} \wedge \psi_{2}\right| \neg \psi|\mathrm{X} \psi| \psi_{1} \mathrm{U} \psi_{2}$ & \\
\hline $\mathrm{PCTL}^{*-}$ & $\varphi\left|\psi_{1} \wedge \psi_{2}\right| \neg \psi \mid \mathrm{X} \psi$ & \\
\hline $\mathrm{PCTL}_{i}^{*-}$ & $\varphi\left|\psi_{1} \wedge \psi_{2}\right| \neg \psi \mid \mathrm{X} \psi$ & Depth $(\psi) \leq i$ \\
\hline $\mathrm{PCTL}^{-}$ & $\mathrm{X} \varphi\left|\varphi_{1} \mathrm{U} \varphi_{2}\right| \varphi_{1} \mathrm{U}^{\leq n} \varphi_{2}$ & \\
\hline $\mathrm{PCTL}^{-}$ & $\mathrm{X} \varphi \mid \varphi_{1} \mathrm{U}^{\leq n} \varphi_{2}$ & \\
\hline $\mathrm{PCTL}_{i}^{-}$ & $\mathrm{X} \varphi \mid \varphi_{1} \mathrm{U}^{\leq j} \varphi_{2}$ & $j \leq i$ \\
\hline
\end{tabular}

3.3. Strong probabilistic bisimulation. In this section we introduce the definition of strong probabilistic bisimulation [35. Let $\left\{s \rightarrow \mu_{i}\right\}_{i \in I}$ be a collection of transitions of $\mathcal{P}$, and let $\left\{w_{i}\right\}_{i \in I}$ be a collection of probabilities with $\sum_{i \in I} w_{i}=1$. Then $\left(s, \sum_{i \in I} w_{i} \cdot \mu_{i}\right)$ is called a combined transition and is denoted by $s \rightarrow_{\mathrm{P}} \mu$ where $\mu=\sum_{i \in I} w_{i} \cdot \mu_{i}$.

Definition 3.3. An equivalence relation $\mathcal{R} \subseteq S \times S$ is a strong probabilistic bisimulation iff $s \mathcal{R} r$ implies that $L(s)=L(r)$ and for each $s \rightarrow \mu$, there exists a combined transition $r \rightarrow \mathrm{P} \mu^{\prime}$ such that $\mu \mathcal{R} \mu^{\prime}$.

We write $s \sim \sim_{\mathrm{P}} r$ whenever there is a strong probabilistic bisimulation $\mathcal{R}$ such that $s \mathcal{R} r$.

It was shown in [35] that $\sim_{\mathrm{P}}$ is preserved by $\|$, that is, $s \sim_{\mathrm{P}} r$ implies $s\left\|t \sim_{\mathrm{P}} r\right\| t$ for any $t$. Also strong probabilistic bisimulation is sound for PCTL which means that if $s \sim_{\mathrm{P}} r$ then for any state formula $\varphi$ of PCTL, $s \models \varphi$ iff $r \models \varphi$. But the other way around is not true, i.e. strong probabilistic bisimulation is not complete for PCTL, as illustrated by the following example.

Example 3.4. Consider again the two PAs in Fig. 1 and assume that all the states have different labels except $s$ and $r$. In addition, $s_{1}, s_{2}$, and $s_{3}$ only have one transition to themselves with probability 1 . The only difference between $s$ and $r$ is that $r$ has an extra step. We can see that the maximal/minimal probabilities from $s$ and $r$ to any set $C \subseteq$ $\left\{s_{1}, s_{2}, s_{3}\right\}$ are the same, therefore it holds that $s \sim \sim_{\mathrm{PCTL}^{*}} r$. But by Definition 3.3 we have $s \nsim_{\mathrm{P}} r$, because the middle transition of $r$ cannot be simulated by $s$ even if we use combined transitions i.e. there is no $a, b \in[0,1]$ such that $0.3 \cdot a+0.5 \cdot b=0.4,0.3 \cdot a+0.4 \cdot b=0.3$, and $0.4 \cdot a+0.1 \cdot b=0.3$. Therefore we conclude that strong probabilistic bisimulation is not complete for $\mathrm{PCTL}^{*}$ as well as for PCTL.

It should be noted that PCTL* distinguishes more states in a PA than PCTL. Refer to the following example.

Example 3.5. Suppose $s$ and $r$ are given by Fig. 1 where each of $s_{1}, s_{2}$, and $s_{3}$ is extended with a transition such that $s_{1} \rightarrow \mu_{1}$ with $\mu_{1}\left(s_{1}\right)=0.6$ and $\mu_{1}\left(s_{4}\right)=0.4, s_{2} \rightarrow \mu_{2}$ with $\mu_{2}\left(s_{4}\right)=1$, and $s_{3} \rightarrow \mu_{3}$ with $\mu_{3}\left(s_{3}\right)=0.5$ and $\mu_{3}\left(s_{4}\right)=0.5$. Here we assume that every state satisfies different atomic propositions except that $L(s)=L(r)$. Then it is not hard to see $s \sim_{\mathrm{PCTL}} r$ while $s \nsim_{\mathrm{PCTL}^{*}} r$. Consider the $\mathrm{PCTL}^{*}$ formula

$$
\varphi=\mathcal{P}_{\leq 0.38}\left(\mathbf{X}\left(L\left(s_{1}\right) \vee L\left(s_{3}\right)\right) \wedge \mathbf{X} \mathbf{X}\left(L\left(s_{1}\right) \vee L\left(s_{3}\right)\right)\right)
$$

it holds $s \models \varphi$ but $r \not \models \varphi$. Note that $\varphi$ is not a well-formed PCTL formula. Indeed, states $s$ and $r$ are PCTL-equivalent. 
We remark that CTL and CTL* equivalences coincide on transition systems, but quantitative properties have more distinguishing power at this point.

We have the following theorem:

\section{Theorem 3.6.}

(1) $\sim_{\mathrm{PCTL}}, \sim_{\mathrm{PCTL}^{*}}, \sim_{\mathrm{PCTL}^{-}}, \sim_{\mathrm{PCTL}_{i}^{-}}, \sim_{\mathrm{PCTL}^{*-}}, \sim_{\mathrm{PCTL}_{i}^{*-}}$, and $\sim_{\mathrm{P}}$ are equivalence relations for any $i \geq 1$.

(2) $\sim_{\mathrm{P}} \subset \sim_{\mathrm{PCTL}^{*}} \subset \sim_{\mathrm{PCTL}}$.

(3) $\sim_{\mathrm{PCTL}^{*-}} \subset \sim_{\mathrm{PCTL}^{-}}$.

(4) $\sim_{\mathrm{PCTL}_{1}^{*-}}=\sim_{\mathrm{PCTL}_{1}^{-}}$

(5) $\sim_{\mathrm{PCTL}_{i}^{*-}} \subset \sim_{\mathrm{PCTL}_{i}^{-}}$for any $i>1$.

(6) $\sim_{\mathrm{PCTL}} \subset \sim_{\mathrm{PCTL}^{-}} \subset \sim_{\mathrm{PCTL}_{i+1}^{-}} \subset \sim_{\mathrm{PCTL}_{i}^{-}}$for all $i \geq 0$.

(7) $\sim_{\mathrm{PCTL}^{*}} \subset \sim_{\mathrm{PCTL}^{*-}} \subset \sim_{\mathrm{PCTL}_{i+1}^{*-}} \subset \sim_{\mathrm{PCTL}_{i}^{*-}}$ for all $i \geq 0$.

Proof. We take $\sim_{\mathrm{PCTL}}$ as an example and all the others can be proved in a similar way. The reflexivity is trivial. If $s \sim \sim_{\mathrm{PCTL}} r$, then we also have $r \sim_{\mathrm{PCTL}} s$ since $s$ and $r$ satisfy the same set of formulas, hence we prove the symmetry of $\sim_{\mathrm{PCTL}}$. Now we prove the transitivity, that is, for any $s, r, t$ if we have $s \sim_{\mathrm{PCTL}} r$ and $r \sim_{\mathrm{PCTL}} t$, then $s \sim_{\mathrm{PCTL}} t$. It is also easy, since $s$ and $r$ satisfy the same set of formulas, and $r$ and $t$ satisfy the same set of formulas by $s \sim_{\mathrm{PCTL}} r$ and $r \sim_{\mathrm{PCTL}} t$, as a result $s \models \varphi$ implies $t \models \varphi$ and vice versa for any $\varphi$, so $s \sim$ PCTL $t$. We conclude that $\sim_{\mathrm{PCTL}}$ is an equivalence relation.

The proof of $\sim_{P} \subset \sim_{P C T L}$ can be found in [35] while $\sim_{P} \subset \sim_{P_{C C T L}}$ can be proved in a similar way. The proof of $\sim_{\mathrm{PCTL}}{ }^{*} \subset \sim_{\mathrm{PCTL}}$ is trivial since PCTL is a subset of PCTL*. Example 3.5 shows that there exists states which are PCTL equivalent, but not $P C T L^{*}$ equivalent, therefore the inclusion is strict.

The proofs of Clause 3 and 5 are obvious since $\sim_{\mathrm{PCTL}}{ }^{-}$is a subset of $\sim_{\mathrm{PCTL}}{ }^{*-}$ while $\sim_{\mathrm{PCTL}_{i}^{-}}$is a subset of $\sim_{\mathrm{PCTL}_{i}^{*-}}$.

We now prove that $\sim_{\mathrm{PCTL}_{1}^{*-}}=\sim_{\mathrm{PCTL}_{1}^{-}}$. It is sufficient to prove that $\mathrm{PCTL}_{1}^{-}$and $\mathrm{PCTL}_{1}^{*-}$ have the same expressiveness. The proof of $\sim_{\mathrm{PCTL}_{1}^{*-}} \subseteq \sim_{\mathrm{PCTL}_{1}^{-}}$is easy since $\mathrm{PCTL}_{1}^{-}$is a subset of $\mathrm{PCTL}_{1}^{*-}$. We now show how formulas of $\mathrm{PCTL}_{1}^{*-}$ can be encoded by formulas of $\mathrm{PCTL}_{1}^{-}$. It is not hard to see that the syntax of path formulas of $\mathrm{PCTL}_{1}^{*-}$ can be rewritten as:

$$
\psi::=\varphi|\mathbf{X} \varphi| \neg \psi \mid \psi_{1} \wedge \psi_{2}
$$

where we replace $\mathrm{X} \psi$ with $\mathrm{X} \varphi$ since $\mathrm{PCTL}_{1}^{*-}$ only allows path formulas whose depths are less or equal than 1. Since $\neg \mathrm{X} \varphi=\mathrm{X} \neg \varphi$, therefore we only need to consider the following cases: $\mathcal{P}_{\bowtie q}(\varphi), \mathcal{P}_{\bowtie q}\left(\mathbf{X} \varphi_{1} \wedge \mathbf{X} \varphi_{2}\right), \mathcal{P}_{\bowtie q}\left(\mathbf{X} \varphi_{1} \wedge \varphi_{2}\right)$, and $\mathcal{P}_{\bowtie q}(\neg \psi)$. We distinguish several cases:

(1) $0<q \leq 1$ and $\bowtie=\geq$ :

(a) $s \models \mathcal{P}_{\geq q}(\varphi)$ iff $s \models \varphi$;

(b) $s \models \mathcal{P}_{\geq q}\left(\mathbf{X} \varphi_{1} \wedge \mathbf{X} \varphi_{2}\right)$ iff $s \models \mathcal{P}_{\geq q}\left(\mathbf{X}\left(\varphi_{1} \wedge \varphi_{2}\right)\right)$;

(c) $s \models \mathcal{P}_{\geq q}\left(\mathbf{X} \varphi_{1} \wedge \varphi_{2}\right)$ iff $s \models \varphi_{2} \wedge \mathcal{P}_{\geq q}\left(\mathbf{X} \varphi_{1}\right)$;

(d) $s \models \mathcal{P}_{\geq q}(\neg \psi)$ iff $s \models \mathcal{P}_{\leq(1-q)}(\psi)$.

(2) $q=0$ and $\bowtie=\geq$ :

This case is trivial, since $s \models \mathcal{P}_{\geq 0}(\psi)$ iff $s \models \top$ for any $\psi$ where $\top=a \vee \neg a$ for some $a$.

(3) $0 \leq q<1$ and $\bowtie=\leq$ :

(a) $s \models \mathcal{P}_{\leq q}(\varphi)$ iff $s \not \models \varphi$; 
(b) $s \models \mathcal{P}_{\leq q}\left(\mathbf{X} \varphi_{1} \wedge \mathbf{X} \varphi_{2}\right)$ iff $s \models \mathcal{P}_{\leq q}\left(\mathbf{X}\left(\varphi_{1} \wedge \varphi_{2}\right)\right)$;

(c) $s \models \mathcal{P}_{\leq q}\left(\mathbf{X} \varphi_{1} \wedge \varphi_{2}\right)$ iff $s \models \neg \varphi_{2} \vee \mathcal{P}_{\leq q}\left(\mathbf{X} \varphi_{1}\right)$;

(d) $s \models \mathcal{P}_{\leq q}(\neg \psi)$ iff $s \models \mathcal{P}_{\geq(1-q)}(\psi)$.

(4) $q=1$ and $\bowtie=\leq$ :

Similar as Clause $(2), s \models \mathcal{P}_{\leq 1}(\psi)$ iff $s \models \top$ for any $\psi$.

(5) The cases when $\bowtie=>$ or $<$ are similar and omitted here.

The proofs of Clauses 6 and 7 are straightforward.

\section{A novel Strong Bisimulation}

This section presents our main contribution of the paper: We introduce a novel notion of strong bisimulation and strong branching bisimulation. We shall show that they agree with PCTL and PCTL* equivalences, respectively. As a preparation step we introduce the strong 1-depth bisimulation.

\subsection{Strong 1-depth bisimulation.}

Definition 4.1. A relation $\mathcal{R} \subseteq S \times S$ is a strong 1-depth bisimulation if $s \mathcal{R} r$ implies that $L(s)=L(r)$ and for any $\mathcal{R}$ downward closed set $C$

(1) for each $s \rightarrow \mu$, there exists $r \rightarrow \mu^{\prime}$ such that $\mu^{\prime}(C) \geq \mu(C)$,

(2) for each $r \rightarrow \mu$, there exists $s \rightarrow \mu^{\prime}$ such that $\mu^{\prime}(C) \geq \mu(C)$.

We write $s \sim_{1} r$ whenever there is a strong 1-depth bisimulation $\mathcal{R}$ such that $s \mathcal{R} r$.

The - though very simple - definition requires only one step matching of the distributions out of $s$ and $r$. The essential difference to the standard definition is: The quantification of the downward closed set comes before the quantification over transition. This is indeed the key of the new definition of bisimulation. The following theorem shows that $\sim_{1}$ agrees with $\sim_{\mathrm{PCTL}_{1}^{-}}$and $\sim_{\mathrm{PCTL}_{1}^{*-}}$ which is also an equivalence relation:

\section{Lemma 4.2. $\sim_{\mathrm{PCTL}_{1}^{-}}=\sim_{1}=\sim_{\mathrm{PCTL}_{1}^{*-}}$.}

Proof. According to Clause (4) of Theorem 3.6, it is enough to prove that $\sim_{\mathrm{PCTL}_{1}^{-}}=\sim_{1}$. Refer to the proof of Theorem 4.7 for the details.

Note that in Definition 4.1 we consider all the $\mathcal{R}$ downward closed sets since it is not enough to only consider $\mathcal{R}$ downward closed sets in $\left\{\mathcal{R}^{\downarrow}(s) \mid s \in S\right\}$. Refer to the following example:

Example 4.3. Suppose there are four absorbing states $s_{1}, s_{2}, s_{3}$, and $s_{4}$ with different atomic propositions, and we have two processes $s$ and $r$ such that $L(s)=L(r)$, and $s \rightarrow \mu_{1}$, $s \rightarrow \mu_{2}, r \rightarrow \nu_{1}, r \rightarrow \nu_{2}$ where $\mu_{1}\left(s_{1}\right)=0.5, \mu_{1}\left(s_{2}\right)=0.5, \mu_{2}\left(s_{3}\right)=0.5, \mu_{2}\left(s_{4}\right)=0.5$, $\nu_{1}\left(s_{1}\right)=0.5, \nu_{1}\left(s_{3}\right)=0.5, \nu_{2}\left(s_{2}\right)=0.5, \nu_{2}\left(s_{4}\right)=0.5$. Let $\mathcal{R}=\{(s, r)\} \cup I D$ where $I D$ denote the identical relation. If we only consider $\mathcal{R}$ downward closed sets in $\left\{\mathcal{R}^{\downarrow}(s) \mid s \in S\right\}$ where $S=\left\{s, r, s_{1}, s_{2}, s_{3}, s_{4}\right\}$, then we will conclude that $s \sim_{1} \quad r$, but $r \models \varphi$ while $s \not \models \varphi$ where $\varphi=\mathcal{P}_{\geq 0.5}\left(\mathbf{X}\left(L\left(s_{1}\right) \vee L\left(s_{2}\right)\right)\right)$.

It turns out that $\sim_{1}$ is preserved by $\|$, implying that $\sim_{\mathrm{PCTL}}^{-}$and $\sim_{\mathrm{PCTL}}^{*-}$ are preserved by $\|$ as well. 
Lemma 4.4. $s \sim_{1} r$ implies that $s\left\|t \sim_{1} r\right\| t$ for any $t$.

Proof. Let $\mathcal{R}=\left\{(s\|t, r\| t) \mid s \sim_{1} r\right\}$, it is enough to show that $\mathcal{R}$ is a strong 1depth bisimulation. Suppose $s \sim_{1} r$, and there exists $s \| t \rightarrow \mu$ such that $\mu(C)>0$ for a $\mathcal{R}$ downward closed set $C$. We need to show that there exists $r \| t \rightarrow \mu^{\prime}$ such that $\mu^{\prime}(C) \geq \mu(C)$. By the definition of $\|$ operator, if $s \| t \rightarrow \mu$, then either $s \rightarrow \mu_{s}$ with $\mu=\mu_{s} \| \mathcal{D}_{t}$, or $t \rightarrow \mu_{t}$ with $\mu=\mathcal{D}_{s} \| \mu_{t}$. We only consider the case when $\mu=\mu_{s} \| \mathcal{D}_{t}$, since the other one is similar. According to the definition of $\mathcal{R}$, for each $\mathcal{R}$ downward closed set $C$, there exists a $\sim_{1}$ downward closed set $C^{\prime}$ such that $\mu(C)=\mu\left(\left\{s^{\prime} \| t \mid s^{\prime} \in C^{\prime}\right\}\right)=\mu_{s}\left(C^{\prime}\right)$. We have known that $s \sim_{1} r$, so for each $s \rightarrow \mu_{s}$ and $C^{\prime}$, there exists $r \rightarrow \mu_{r}$ such that $\mu_{r}\left(C^{\prime}\right) \geq \mu_{s}\left(C^{\prime}\right)$. Therefore for each $C$ and $s \| t \rightarrow \mu$, there exists $r \| t \rightarrow \mu^{\prime}$ such that

$$
\mu^{\prime}(C)=\mu^{\prime}\left(\left\{s^{\prime}|| t \mid s^{\prime} \in C^{\prime}\right\}\right)=\mu_{r}\left(C^{\prime}\right) \geq \mu_{s}\left(C^{\prime}\right)=\mu\left(\left\{s^{\prime} \| t \mid s^{\prime} \in C^{\prime}\right\}\right)=\mu(C) .
$$

A few remarks are in order.

(1) Note in Definition 5 of [37] we require that $\mathcal{R}$ is a preorder, while the $\mathcal{R}$ in Definition 4.1 can be any relation, we could also have required $\mathcal{R}$ being an equivalence relation: but all of them will induce the same largest bisimulation equivalence.

Bisimulation relations are defined for arbitrary relations for classical transition systems [27]. However, in the literature of bisimulation relations for probabilistic systems, bisimulation relations are defined mostly only for equivalence relations, see for example [26, 25, 34, 3, 35, 28]. For probabilistic systems, defining bisimulations for equivalence relations has the advantage of being very easy to understand. On the other side, a general definition for all relations is important as well, as it particularly sheds light to the connections to the classical transition systems. To the best of our knowledge, such general bisimulation definitions are first defined, independently, in [12, 10, 39]. Later in [29, 20, 32, 17, this general definition has been exploited to study logical characterizations, and characterizing formulas for probabilistic systems.

(2) We note that for Kripke structures (PAs with only Dirac distributions) $\sim_{1}$ agrees with the usual strong bisimulation by Milner [27] if we consider state-labelled instead of transition-labelled systems.

4.2. Strong branching bisimulation. Now we extend the relation $\sim_{1}$ to strong $i$-step bisimulation. Then, the intersection of all of these relations gives us the new notion of strong branching bisimulation, which is shown to be the same as $\sim_{\mathrm{PCTL}}$. Recall that Theorem 3.6 states that $\sim_{\mathrm{PCTL}}$ is strictly coarser than $\sim_{\mathrm{PCTL}}$, which we shall consider in the next section.

Following the approach in [38], we define $\operatorname{Prob}_{\sigma, s}\left(C, C^{\prime}, n, \omega\right)$ which denotes the probability from $s$ to states in $C^{\prime}$ via states in $C$ possibly in at most $n$ steps under scheduler $\sigma$, where $\omega$ is used to keep track of the path and only deterministic schedulers are considered in the following. Formally, $\operatorname{Prob}_{\sigma, s}\left(C, C^{\prime}, n, \omega\right)$ equals 1 if $s \in C^{\prime}$, and else if $n>0 \wedge\left(s \in C \backslash C^{\prime}\right)$, then

$$
\operatorname{Prob}_{\sigma, s}\left(C, C^{\prime}, n, \omega\right)=\sum_{r \in \operatorname{supp}\left(\mu^{\prime}\right)} \mu^{\prime}(r) \cdot \operatorname{Prob}_{\sigma, r}\left(C, C^{\prime}, n-1, \omega r\right) .
$$

where $\sigma(\omega)\left(s, \mu^{\prime}\right)=1$, otherwise $\operatorname{Prob}_{\sigma, s}\left(C, C^{\prime}, n, \omega\right)$ equals 0 , provided $n>0$.

Strong $i$-depth branching bisimulation is a straightforward extension of strong 1-depth bisimulation, where instead of considering only one immediate step, we consider up to $i$ steps. We let $\sim_{1}^{\mathrm{b}}=\sim_{1}$ in the following. 
Definition 4.5. A relation $\mathcal{R} \subseteq S \times S$ is a strong $i$-depth branching bisimulation with $i>1$ if $s \mathcal{R} r$ implies $s \sim_{i-1}^{\mathrm{b}} r$ and for any $\mathcal{R}$ downward closed sets $C, C^{\prime}$,

(1) for each scheduler $\sigma$, there exists a scheduler $\sigma^{\prime}$ such that

$$
\operatorname{Prob}_{\sigma^{\prime}, r}\left(C, C^{\prime}, i, r\right) \geq \operatorname{Prob}_{\sigma, s}\left(C, C^{\prime}, i, s\right),
$$

(2) for each scheduler $\sigma$, there exists a scheduler $\sigma^{\prime}$ such that

$$
\operatorname{Prob}_{\sigma^{\prime}, s}\left(C, C^{\prime}, i, s\right) \geq \operatorname{Prob}_{\sigma, r}\left(C, C^{\prime}, i, r\right) .
$$

We write $s \sim_{i}^{\mathrm{b}} r$ whenever there is a strong $i$-depth branching bisimulation $\mathcal{R}$ such that $s \mathcal{R} r$. The strong branching bisimulation $\sim^{\mathrm{b}}$ is defined as $\sim^{\mathrm{b}}=\cap_{i \geq 1} \sim_{i}^{\mathrm{b}}$.

Obviously, if $\mathcal{R}$ is symmetric, the second condition can be dropped. The following lemma shows that $\sim_{i}^{\mathrm{b}}$ is an equivalence relation, and moreover, $\sim_{i}^{\mathrm{b}}$ decreases until a fixed point is reached.

\section{Lemma 4.6.}

(1) $\sim^{\mathrm{b}}$ and $\sim_{i}^{\mathrm{b}}$ are equivalence relations for any $i \geq 1$.

(2) $\sim_{j}^{\mathrm{b}} \subseteq \sim_{i}^{\mathrm{b}}$ provided that $1 \leq i \leq j$.

(3) There exists $i \geq 1$ such that $\sim_{j}^{\mathrm{b}}=\sim_{k}^{\mathrm{b}}$ for any $j, k \geq i$.

Proof. We only show the proof of transitivity of $\sim_{i}^{\mathrm{b}}$. Suppose that $s \sim_{i}^{\mathrm{b}} t$ and $t \sim_{i}^{\mathrm{b}} r$, we need to prove that $s \sim_{i}^{\mathrm{b}} r$. By Definition 4.5, we know there exists strong $i$-depth branching bisimulations $\mathcal{R}_{1}$ and $\mathcal{R}_{2}$ such that $s \mathcal{R}_{1} t$ and $t \mathcal{R}_{2} r$. Assume $\mathcal{R}_{1}$ and $\mathcal{R}_{2}$ are reflexive, such relations always exist since each state is strong $i$-depth bisimilar to itself. Let $\mathcal{R}=\mathcal{R}_{1} \circ \mathcal{R}_{2}=\left\{\left(s_{1}, s_{3}\right) \mid \exists s_{2} \cdot\left(s_{1} \mathcal{R}_{1} s_{2} \wedge s_{2} \mathcal{R}_{2} r\right)\right\}$, it is enough to show that $\mathcal{R}$ is a strong $i$-depth branching bisimulation. Note $\mathcal{R}_{1} \cup \mathcal{R}_{2} \subseteq \mathcal{R}$, since for each $s_{1} \mathcal{R}_{1} s_{2}$ we also have $s_{2} \mathcal{R}_{2} s_{2}$ due to reflexivity, thus $s_{1} \mathcal{R} s_{2}$, implying $\mathcal{R}_{1} \subseteq \mathcal{R}$. Similarly we can show that $\mathcal{R}_{2} \subseteq \mathcal{R}$. Therefore for any $\mathcal{R}$ downward closed sets $C$ and $C^{\prime}$, they are also $\mathcal{R}_{1}$ and $\mathcal{R}_{2}$ downward closed. Therefore for each $\sigma$, there exists $\sigma^{\prime}$ such that $\operatorname{Prob}_{\sigma^{\prime}, t}\left(C, C^{\prime}, i, t\right) \geq \operatorname{Prob}_{\sigma, s}\left(C, C^{\prime}, i, s\right)$. Since $t \sim_{i}^{\mathrm{b}} r$, thus there exists $\sigma^{\prime \prime}$ such that $\operatorname{Prob}_{\sigma^{\prime \prime}, r}\left(C, C^{\prime}, i, r\right) \geq \operatorname{Prob}_{\sigma^{\prime}, t}\left(C, C^{\prime}, i, t\right) \geq \operatorname{Prob}_{\sigma, s}\left(C, C^{\prime}, i, s\right)$. This completes the proof of transitivity.

The proof of Clause (2) is straightforward from Definition 4.5, since $s \quad \sim_{j}^{\mathrm{b}} r$ implies $s \sim_{j-1}^{\mathrm{b}} r$ when $j>1$.

From Definition 4.5, we can see that $\sim_{i}^{\mathrm{b}}$ is getting more discriminating as $i$ increases. Moreover, in a PA only with finitely many states the maximum number of equivalence classes is equal to the number of states, as result we can guarantee that $\sim_{n}^{\mathrm{b}}=\sim^{\mathrm{b}}$ where $n$ is the total number of states.

Given a relation $\mathcal{R}$, two paths $\omega_{1}=s_{0} s_{1} \ldots, \omega_{2}=r_{0} r_{1} \ldots$ are in $\mathcal{R}$, written as $\omega_{1} \mathcal{R} \omega_{2}$, iff $\omega_{1}[j] \mathcal{R} \omega_{2}[j]$ for all $j \geq 0$. We then define the $\mathcal{R}$ closed paths: The set $\Omega$ of paths is $\mathcal{R}$ closed if for any $\omega_{1} \in \Omega$ and $\omega_{2}$ such that $\omega_{1} \mathcal{R} \omega_{2}$, it holds that $\omega_{2} \in \Omega$. Let $\mathcal{B}_{\mathcal{R}}=\{\Omega \subseteq \mathcal{B} \mid \Omega$ is $\mathcal{R}$ closed $\}$. By standard measure theory $\mathcal{B}_{\mathcal{R}}$ is measurable.

In the following, we will use $\operatorname{Sat}(\varphi)=\{s \in S \mid s \models \varphi\}$ to denote the set of states which satisfy $\varphi$. Similarly, $\operatorname{Sat}(\psi)=\{\omega \in$ Path $\mid \omega \models \psi\}$ is the set of paths satisfying $\psi$. Below we show that $\sim_{i}^{\mathrm{b}}$ characterizes $\mathrm{PCTL}_{i}^{-}$. Moreover, we show that $\sim^{\mathrm{b}}$ agrees with PCTL equivalence.

Theorem 4.7. $\sim_{\mathrm{PCTL}_{i}^{-}}=\sim_{i}^{\mathrm{b}}$ for any $i \geq 1$, moreover $\sim_{\mathrm{PCTL}}=\sim^{\mathrm{b}}$. 
Proof.

(1) $\sim_{\mathrm{PCTL}_{i}^{-}} \subseteq \sim_{i}^{\mathrm{b}}$ :

Let $\mathcal{R}=\left\{(s, r) \mid s \sim_{\mathrm{PCTL}_{i}^{-}} r\right\}$ and $s \mathcal{R} r$. Thus, $\mathcal{R}$ is symmetric. We show that for any $\mathcal{R}$ closed sets $C, C^{\prime}$ and scheduler $\sigma$, there exists a scheduler $\sigma^{\prime}$ such that $\operatorname{Prob}_{\sigma^{\prime}, r}\left(C, C^{\prime}, i, r\right) \geq \operatorname{Prob}_{\sigma, s}\left(C, C^{\prime}, i, s\right)$. Suppose there are $n$ different equivalence classes of $\mathcal{R}$ in a finite PA. Let $\varphi_{C_{i}, C_{j}}$ be a state formula such that $\operatorname{Sat}\left(\varphi_{C_{i}, C_{j}}\right) \supseteq C_{i}$ and $\operatorname{Sat}\left(\varphi_{C_{i}, C_{j}}\right) \cap C_{j}=\emptyset$, here $1 \leq i \neq j \leq n$ and $C_{i}, C_{j} \in S / \mathcal{R}$ are two different equivalence classes. Formula like $\varphi_{C_{i}, C_{j}}$ always exists, otherwise there will not exist a formula which is fulfilled by states in $C_{i}$, but not fulfilled by states in $C_{j}$, that is, states in $C_{i}$ and $C_{j}$ satisfy the same set of formulas, this is against the assumption that $C_{i}$ and $C_{j}$ are two different equivalence classes. Let $\varphi_{C_{i}}={\underset{1 \leq j \neq i \leq n}{\wedge}}_{\varphi_{C_{i}, C_{j}}}$, it is not hard to see that $\operatorname{Sat}\left(\varphi_{C_{i}}\right)=C_{i}$. For a $\mathcal{R}$ closed set $C$ which is a set of equivalence classes, let $\varphi_{C}=$ $\underset{C^{\prime} \in S / \mathcal{R} \wedge C^{\prime} \subseteq C}{\vee} \varphi_{C^{\prime}}$, then it holds $\operatorname{Sat}\left(\varphi_{C}\right)=C$. Now suppose $\operatorname{Prob}_{\sigma, s}\left(C, C^{\prime}, i, s\right)=q$, then we know $s \models \neg \mathcal{P}_{<q}(\psi)$ where $\psi=\varphi_{C} \mathrm{U}^{\leq i} \varphi_{C^{\prime}}$. By assumption $r \models \neg \mathcal{P}_{<q}(\psi)$, so there exists a scheduler $\sigma^{\prime}$ such that $\operatorname{Prob}_{\sigma^{\prime}, r}\left(C, C^{\prime}, i, r\right) \geq q$, that is, $\operatorname{Prob}_{\sigma^{\prime}, r}\left(C, C^{\prime}, i, r\right) \geq$ $\operatorname{Prob}_{\sigma, s}\left(C, C^{\prime}, i, s\right)$.

(2) $\sim_{i}^{\mathrm{b}} \subseteq \sim_{\mathrm{PCTL}_{i}^{-}}:$

The proof is done by structural induction on the syntax of state formula $\varphi$ and path formula $\psi$ of $\mathrm{PCTL}_{i}^{-}$, that is, we need to prove the following two results simultaneously.

(a) $s \sim_{i}^{\mathrm{b}} r$ implies that $s \models \varphi$ iff $r \models \varphi$ for any state formula $\varphi$ of $\mathrm{PCTL}_{i}$.

(b) $\omega_{1} \sim_{i}^{\text {b }} \omega_{2}$ implies that $\omega_{1} \models \psi$ iff $\omega_{2} \models \psi$ for any path formula $\psi$ of $\mathrm{PCTL}_{i}$.

We only consider $\varphi=\mathcal{P}_{\leq q}(\psi)$ where $\psi=\varphi_{1} \mathrm{U}^{\leq i} \varphi_{2}$, since other cases are similar. According to the semantics $s \models \varphi$ iff $\forall \sigma . \operatorname{Prob}_{\sigma, s}(\{\omega \mid \omega \models \psi\}) \leq q$. Since $\psi=\varphi_{1} \mathrm{U}^{\leq i} \varphi_{2}$, we only need to consider prefix of length $i$ for each path. By induction hypothesis $\{\omega|\omega|=\psi\}$ is $\sim_{i}^{\mathrm{b}}$ closed. Since $\psi=\varphi_{1} \mathrm{U}^{\leq i} \varphi_{2}$, there exists $\Omega$ such that $l(\Omega) \leq i$ and $C_{\Omega}=\{\omega \mid \omega \models \psi\}$. According to the semantics of $\mathrm{U}^{\leq i}$, there exists two $\sim_{i}^{\mathrm{b}}$ closed sets $C, C^{\prime}$ such that $\Omega=\underset{0 \leq k<i}{\cup} C^{k} C^{\prime}$. We prove by contradiction, and assume $s \models \varphi$ and $r \not \forall \varphi$. Then for any $\sigma$, we have $\operatorname{Prob}_{\sigma, s}\left(C_{\Omega}\right) \leq q$. Since $r \not \forall \varphi$, there exists $\sigma^{\prime}$ such that $\operatorname{Prob}_{\sigma^{\prime}, r}\left(C_{\Omega}\right)>q$, thus there does not exist $\sigma$ such that $\operatorname{Prob}_{\sigma, s}\left(C, C^{\prime}, i, s\right) \geq$ $\operatorname{Prob}_{\sigma^{\prime}, r}\left(C, C^{\prime}, i, r\right)$, which contradicts the assumption $s \sim_{i}^{\mathrm{b}} r$. Therefore $r \models \varphi$, and $s \sim \sim_{\mathrm{PCTL}_{i}^{-}} r$.

(3) $\sim_{\mathrm{PCTL}} \subseteq \sim^{\mathrm{b}}$ :

Since $\sim_{\mathrm{PCTL}} \subseteq \sim_{\mathrm{PCTL}_{i}^{-}}$for each $i \geq 0$, thus $\sim_{\mathrm{PCTL}} \subseteq \cap_{i \geq 0} \sim_{\mathrm{PCTL}_{i}^{-}}$. According to above proof, $\sim_{\mathrm{PCTL}} \subseteq \cap_{i \geq 0} \sim_{\mathrm{PCTL}_{i}^{-}}=\cap_{i \geq 0} \sim_{i}^{\mathrm{b}}=\sim^{\mathrm{b}}$.

(4) $\sim^{\mathrm{b}} \subseteq \sim_{\mathrm{PCTL}}:$

For any scheduler $\sigma$, we have

$$
\operatorname{Prob}_{\sigma, s}\left(\left\{\omega \mid \omega \models \varphi_{1} \cup \leq i \varphi_{2}\right\}\right)=\operatorname{Prob}_{\sigma, s}\left(\operatorname{Sat}\left(\varphi_{1}\right), \operatorname{Sat}\left(\varphi_{2}\right), i, s\right) .
$$

Moreover $\operatorname{Prob}_{\sigma, s}\left(\left\{\omega \mid \omega \models \varphi_{1} \cup \varphi_{2}\right\}\right)=\lim _{i \rightarrow \infty} \operatorname{Prob}_{\sigma, s}\left(\operatorname{Sat}\left(\varphi_{1}\right), \operatorname{Sat}\left(\varphi_{2}\right), i, s\right)$, the limit exists since $\left\{\operatorname{Prob}_{\sigma, s}\left(\operatorname{Sat}\left(\varphi_{1}\right), \operatorname{Sat}\left(\varphi_{2}\right), i, s\right) \mid i \geq 0\right\}$ is a monotonic and bounded i.e. convergent sequence. Therefore $s \sim_{\mathrm{PCTL}_{i}^{-}} r\left(\right.$ or $\left.s \sim_{i}^{\mathrm{b}} r\right)$ for any $i \geq 0$ implies that $s \sim \mathrm{PCTL} r$. 


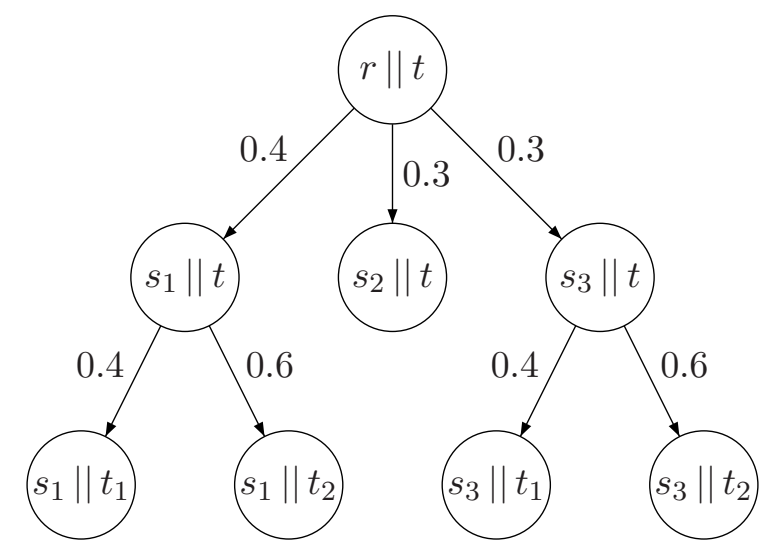

Figure 2: $\sim_{i}^{\mathrm{b}}$ is not compositional when $i>1$

Intuitively, since $\sim_{i}^{\mathrm{b}}$ becomes smaller as $i$ increases, for any PA, $\sim_{i}^{\mathrm{b}}$ will eventually converge to PCTL equivalence.

Recall $\sim_{1}^{\mathrm{b}}$ is compositional by Lemma 4.4, which unfortunately is not the case for $\sim_{i}^{\mathrm{b}}$ with $i>1$. This is illustrated by the following example:

Counterexample 1. $s \sim_{i}^{\mathrm{b}} r$ does not necessarily imply $s\left\|t \sim_{i}^{\mathrm{b}} r\right\| t$ for any $t$ generally if $i>1$.

We have shown in Example 3.4 that $s \sim$ PCTL $r$. If we compose $s$ and $r$ with $t$ where $t$ only has a transition to $\mu$ such that $\mu\left(t_{1}\right)=0.4$ and $\mu\left(t_{2}\right)=0.6$, then it turns out that $s\left\|t \nsim_{\mathrm{PCTL}} r\right\| t$. Since there exists $\varphi=\mathcal{P}_{\leq 0.34}(\psi)$ with

$$
\psi=\left(\left(L(s \| t) \vee L\left(s_{1} \| t\right) \vee\left(L\left(s_{3} \| t\right)\right)\right) \bigcup^{\leq 2}\left(L\left(s_{1} \| t_{2}\right) \vee L\left(s_{3} \| t_{1}\right)\right)\right)
$$

such that $s \| t \models \varphi$ but $r \| t \not \models \varphi$, as there exists a scheduler $\sigma$ such that the probability of paths satisfying $\psi$ in $\operatorname{Prob}_{\sigma, r}$ equals 0.36 . Fig. 2 shows the execution of $r \| t$ guided by $\sigma$, where we assume all the states in Fig. 2 have different atomic propositions except that $L(s \| t)=L(r \| t)$. It is similar for $\sim_{\mathrm{PCT}^{*}}$.

Note that $\varphi$ is also a well-formed state formula of $\mathrm{PCTL}_{2}^{-}$, so $\sim_{\mathrm{PCTL}_{i}^{-}}$as well as $\sim_{i}^{\mathrm{b}}$ are not compositional if $i \geq 2$.

4.3. Strong bisimulation. In this section we introduce a new notion of strong bisimulation and show that it characterizes $\sim \sim_{\mathrm{PCTL}}{ }^{*}$. Given a relation $\mathcal{R}$, a $\mathcal{R}$ downward closed cone $C_{\Omega}$ and a measure $\operatorname{Prob}$, the value of $\operatorname{Prob}\left(C_{\Omega}\right)$ can be computed by summing up the values of all $\operatorname{Prob}\left(C_{\omega}\right)$ with $\omega \in \Omega$. We let $\widetilde{\Omega} \subseteq\left(\mathcal{R}^{\downarrow}\right)^{+}$be a set of $\mathcal{R}$ downward closed paths, then $C_{\widetilde{\Omega}}$ is the corresponding set of $\mathcal{R}$ downward closed cones, that is, $C_{\widetilde{\Omega}}=\cup_{\Omega \in \widetilde{\Omega}} C_{\Omega}$. Define $l(\widetilde{\Omega})=\operatorname{Max}\{l(\Omega) \mid \Omega \in \widetilde{\Omega}\}$ as the maximum length of $\Omega$ in $\widetilde{\Omega}$. To compute $\operatorname{Prob}\left(C_{\widetilde{\Omega}}\right)$, we cannot sum up the value of each $\operatorname{Prob}\left(C_{\Omega}\right)$ such that $\Omega \in \widetilde{\Omega}$ as before, since we may have a path $\omega$ such that $\omega \in \Omega_{1}$ and $\omega \in \Omega_{2}$ where $\Omega_{1}, \Omega_{2} \in \widetilde{\Omega}$, so we have to remove these duplicate paths and make sure each path is considered once and only once as follows, where we abuse the notation and write $\omega \in \widetilde{\Omega}$ iff $\exists \Omega \in \widetilde{\Omega} . \omega \in \Omega$ :

$$
\operatorname{Prob}\left(C_{\widetilde{\Omega}}\right)=\sum_{\omega \in \widetilde{\Omega} \wedge \exists \omega^{\prime} \in \widetilde{\Omega} \cdot \omega^{\prime} \leq \omega} \operatorname{Prob}\left(C_{\omega}\right)
$$


Note Equation 4.2 can be extended to compute the probability of any set of cones in a given measure.

The definition of strong $i$-depth bisimulation is as follows:

Definition 4.8. A relation $\mathcal{R} \subseteq S \times S$ is a strong $i$-depth bisimulation if $i>1$ and $s \mathcal{R} r$ implies that $s \sim_{i-1} r$ and for any $\widetilde{\Omega} \subseteq\left(\mathcal{R}^{\downarrow}\right)^{+}$with $l(\widetilde{\Omega})=i$

(1) for each scheduler $\sigma$, there exists $\sigma^{\prime}$ such that $\operatorname{Prob}_{\sigma^{\prime}, r}\left(C_{\widetilde{\Omega}}\right) \geq \operatorname{Prob}_{\sigma, s}\left(C_{\widetilde{\Omega}}\right)$,

(2) for each scheduler $\sigma$, there exists $\sigma^{\prime}$ such that $\operatorname{Prob}_{\sigma^{\prime}, s}\left(C_{\widetilde{\Omega}}\right) \geq \operatorname{Prob}_{\sigma, r}\left(C_{\widetilde{\Omega}}\right)$.

We write $s \sim_{i} r$ whenever there is a $i$-depth strong bisimulation $\mathcal{R}$ such that $s \mathcal{R} r$. The strong bisimulation $\sim$ is defined as $\sim=\cap_{i \geq 1} \sim_{i}$.

Recall that $\left(\mathcal{R}^{\downarrow}\right)^{+}$contains all the downward closed paths. Each downward closed path can be equivalently stated as a sequence of downward closed sets, thus Definition 4.8 subsumes Definition 4.5 in the sense that the two downward closed sets $C$ and $C^{\prime}$ in Definition 4.5 can be seen as a special downward closed path of form $C C \ldots C^{\prime}$. Similar to $\sim_{i}^{\mathrm{b}}$, the relation $\sim_{i}$ forms a chain of equivalence relations, and $\sim_{i}$ will converge finally in a PA.

\section{Lemma 4.9.}

(1) $\sim_{i}$ is an equivalence relation for any $i>1$.

(2) $\sim_{j} \subseteq \sim_{i}$ provided that $1 \leq i \leq j$

(3) There exists $i \geq 1$ such that $\sim_{j}=\sim_{k}$ for any $j, k \geq i$.

Proof. For the first clause we only prove the transitivity since the reflexivity and symmetry are easy. Suppose that $s \sim_{i} r$ and $r \sim_{i} t$, we need to show that $s \sim_{i} t$. According to Definition 4.8, we know there exists strong $i$-depth bisimulations $\mathcal{R}_{1}$ and $\mathcal{R}_{2}$ such that $s \mathcal{R}_{1} t$ and $t \mathcal{R}_{2} r$. Let $\mathcal{R}=\mathcal{R}_{1} \circ \mathcal{R}_{2}=\left\{\left(s_{1}, s_{3}\right) \mid \exists s_{2} .\left(s_{1} \mathcal{R}_{1} s_{2} \wedge s_{2} \mathcal{R}_{2} r\right)\right\}$, it is enough to show that $\mathcal{R}$ is a strong $i$-depth bisimulation. Similar as in the proof of Lemma 4.6, if $\widetilde{\Omega} \subseteq\left(\mathcal{R}^{\downarrow}\right)^{+}$, then it also holds that $\widetilde{\Omega} \subseteq\left(\mathcal{R}_{1}^{\downarrow}\right)^{+}$and $\widetilde{\Omega} \subseteq\left(\mathcal{R}_{2}^{\downarrow}\right)^{+}$. Thus for each $\widetilde{\Omega} \subseteq\left(\mathcal{R}^{\downarrow}\right)^{+}$ with $l(\widetilde{\Omega})=i$, and scheduler $\sigma$ of $s$, there exists $\sigma^{\prime}$ of $r$ such that $\operatorname{Prob}_{\sigma^{\prime}, r}(\widetilde{\Omega}) \geq \operatorname{Prob}_{\sigma, s}(\widetilde{\Omega})$. Since $r \sim_{i} t$, there exists scheduler $\sigma^{\prime \prime}$ of $t$ such that

$$
\operatorname{Prob}_{\sigma^{\prime \prime}, t}(\widetilde{\Omega}) \geq \operatorname{Prob}_{\sigma^{\prime}, r}(\widetilde{\Omega}) \geq \operatorname{Prob}_{\sigma, s}(\widetilde{\Omega}) .
$$

The other direction is similar and omitted here, thus $s \sim_{i} t$.

The proof for the second clause is straightforward from Definition 4.8. For the last one, since there are only finitely many states, thus there are only finitely many equivalence classes, such $i$ always exists.

Below we show that $\sim_{i}$ characterizes $\sim_{\mathrm{PCTL}_{i}^{*-}}$ for all $i \geq 1$, where $\sim=\underset{n \geq 1}{\cap} \sim_{n}$.

Theorem 4.10. $\sim_{\mathrm{PCTL}_{i}^{*-}}=\sim_{i}$ for any $i \geq 1$, moreover $\sim_{\mathrm{PCTL}^{*}}=\sim$.

Proof.

(1) $\sim_{\mathrm{PCTL}_{i}^{*-}} \subseteq \sim_{i}:$

Let $\mathcal{R}=\left\{(s, r) \mid s \sim_{\mathrm{PCTL}_{i}^{*-}} r\right\}$ and $s \mathcal{R} r$, obviously $\mathcal{R}$ is symmetric. We show that for any $\widetilde{\Omega} \subseteq\left(\mathcal{R}^{\downarrow}\right)^{+}$with $l(\widetilde{\Omega})=i$ and scheduler $\sigma$, there exists a scheduler $\sigma^{\prime}$ such that $\operatorname{Prob}_{\sigma^{\prime}, r}\left(C_{\widetilde{\Omega}}\right) \geq \operatorname{Prob}_{\sigma, s}\left(C_{\widetilde{\Omega}}\right)$. Following the way in the proof of Theorem 4.7, we can construct a formula $\varphi_{C}$ such that $\operatorname{Sat}\left(\varphi_{C}\right)=C$ where $C$ is a $\mathcal{R}$ closed set. Suppose $\Omega=C_{0} C_{1} \ldots C_{j}$ with $j \leq i$, then

$$
\psi_{\Omega}=\varphi_{C_{0}} \wedge \mathrm{X}\left(\varphi_{C_{1}} \wedge \ldots \wedge \mathrm{X}\left(\varphi_{C_{j-1}} \wedge \mathrm{X} \varphi_{C_{j}}\right) \ldots\right)
$$


can be used to characterize $\Omega$, that is, $\operatorname{Sat}\left(\psi_{\Omega}\right)=C_{\Omega}$. Let $\psi=\underset{\Omega \in \widetilde{\Omega}}{\vee} \psi_{\Omega}$, then $\operatorname{Sat}(\psi)=$ $C_{\widetilde{\Omega}}$. As a result $s \models \neg \mathcal{P}_{<q}(\psi)$ where $q=\operatorname{Prob}_{\sigma, s}\left(C_{\widetilde{\Omega}}\right)$. By assumption $r \models \neg \mathcal{P}_{<q}(\psi)$, so there exists a scheduler $\sigma^{\prime}$ such that $\operatorname{Prob}_{\sigma^{\prime}, r}\left(C_{\widetilde{\Omega}}\right) \geq q$, that is, $\operatorname{Prob}_{\sigma^{\prime}, r}\left(C_{\widetilde{\Omega}}\right) \geq$ $\operatorname{Prob}_{\sigma, s}\left(C_{\widetilde{\Omega}}\right)$.

(2) $\sim_{i} \subseteq \sim_{\mathrm{PCTL}_{i}^{*-}}$ :

The proof is by structural induction on the syntax of state formula $\varphi$ and path formula $\psi$ of $\mathrm{PCTL}_{i}^{*-}$, that is, we need to prove the following two results simultaneously.

(a) $s \sim_{i} r$ implies that $s \models \varphi$ iff $r \models \varphi$ for any state formula $\varphi$ of $\mathrm{PCTL}_{i}^{*-}$.

(b) $\omega_{1} \sim_{i} \omega_{2}$ implies that $\omega_{1} \models \psi$ iff $\omega_{2} \models \psi$ for any path formula $\psi$ of $\mathrm{PCTL}_{i}^{*-}$.

We only consider $\varphi=\mathcal{P}_{\leq q}(\psi)$ such that $\operatorname{Depth}(\psi) \leq i$. By induction hypothesis $\{\omega \mid$ $\omega \models \psi\}$ is $\sim_{i}$ closed. Since $\operatorname{Depth}(\psi) \leq i$, there exists $\widetilde{\Omega}$ such that $l(\widetilde{\Omega}) \leq i$ and $C_{\widetilde{\Omega}}=\{\omega \mid \omega \models \psi\}$. We prove by contradiction, and assume that $s \models \varphi$ and $r \not \models \varphi$. According to the semantics $s \models \varphi$ iff $\forall \sigma \cdot \operatorname{Prob}_{\sigma, s}\left(C_{\widetilde{\Omega}}\right) \leq q$. If $r \not \models \varphi$, then there exists $\sigma^{\prime}$ such that $\operatorname{Prob}_{\sigma^{\prime}, r}\left(C_{\widetilde{\Omega}}\right)>q$, consequently for such $\sigma^{\prime}$ of $r$ there does not exist $\sigma$ of $s$ such that $\operatorname{Prob}_{\sigma, s}\left(C_{\widetilde{\Omega}}\right) \geq \operatorname{Prob}_{\sigma^{\prime}, r}\left(C_{\widetilde{\Omega}}\right)$ which contradicts the assumption that $s \sim_{i} r$, therefore $r \models \varphi$ and $s \sim_{\mathrm{PCTL}_{i}^{*-}} r$.

(3) $\sim_{\mathrm{PCTL}^{*}}=\sim$ :

The arguments are similar as in Theorem 4.7

For the same reason as strong $i$-depth branching bisimulation, $\sim_{i}$ is not preserved by $\|$ when $i>1$.

Counterexample 2. $s \sim_{i} r$ does not necessarily imply $s\left\|t \sim_{i} r\right\| t$ for any $t$ generally if $i>1$. This can be shown by using the same arguments as in Counterexample 1,

4.4. Taxonomy for strong bisimulations. Fig. 3 summaries the relationship among all these bisimulations and logical equivalences, where $\rightarrow$ denotes $\subseteq$ and $\nrightarrow$ denotes $\not$. We also abbreviate $\sim$ PCTL as PCTL, and similarly for other logical equivalences. The parameter $n$ means that for any finite PA, there always exists a $n \geq 0$ such that $\sim_{\mathrm{PCTL}_{n}^{-}}=\sim_{n}^{\mathrm{b}}$ and $\sim_{\mathrm{PCTL}_{n}^{*-}}=\sim_{n}$. Congruent relations with respect to the $\|$ operator are shown in circles, and non-congruent relations are shown in boxes. Segala and Lynch have considered another strong bisimulation in 35, which can be defined by replacing the $r \rightarrow_{\mathrm{p}} \mu^{\prime}$ with $r \rightarrow \mu^{\prime}$ in Definition 3.3 and thus is strictly stronger than $\sim_{p}$. It is also worth mentioning that all the bisimulations shown in Fig. 3 coincide with the strong bisimulation defined in [5] in the DTMCs setting (PAs without non-deterministic choices).

\section{WEAK BISIMULATIONS}

As in [5] we use PCTL $\backslash x$ to denote the subset of PCTL without the next operator $\mathrm{X} \varphi$ and the bounded until $\varphi_{1} \mathrm{U}^{\leq n} \varphi_{2}$. Similarly, $\mathrm{PCTL}{ }_{\backslash \mathrm{X}}^{*}$ is used to denote the subset of PCTL* without the next operator $\mathbf{X} \psi$. In this section we shall introduce weak bisimulations and study their relation to $\sim_{\mathrm{PCTL}} \mathrm{X}_{\mathrm{X}}$ and $\sim_{\mathrm{PCTL}}{ }_{\backslash \mathrm{X}}^{*}$, respectively. Before this we should point out that $\sim_{\mathrm{PCTL}}{ }_{\backslash \mathrm{X}}^{*}$ implies $\sim \sim_{\mathrm{PCTL}} \mathrm{X}_{\mathrm{X}}$ but the other direction does not hold. Refer to the following example. 


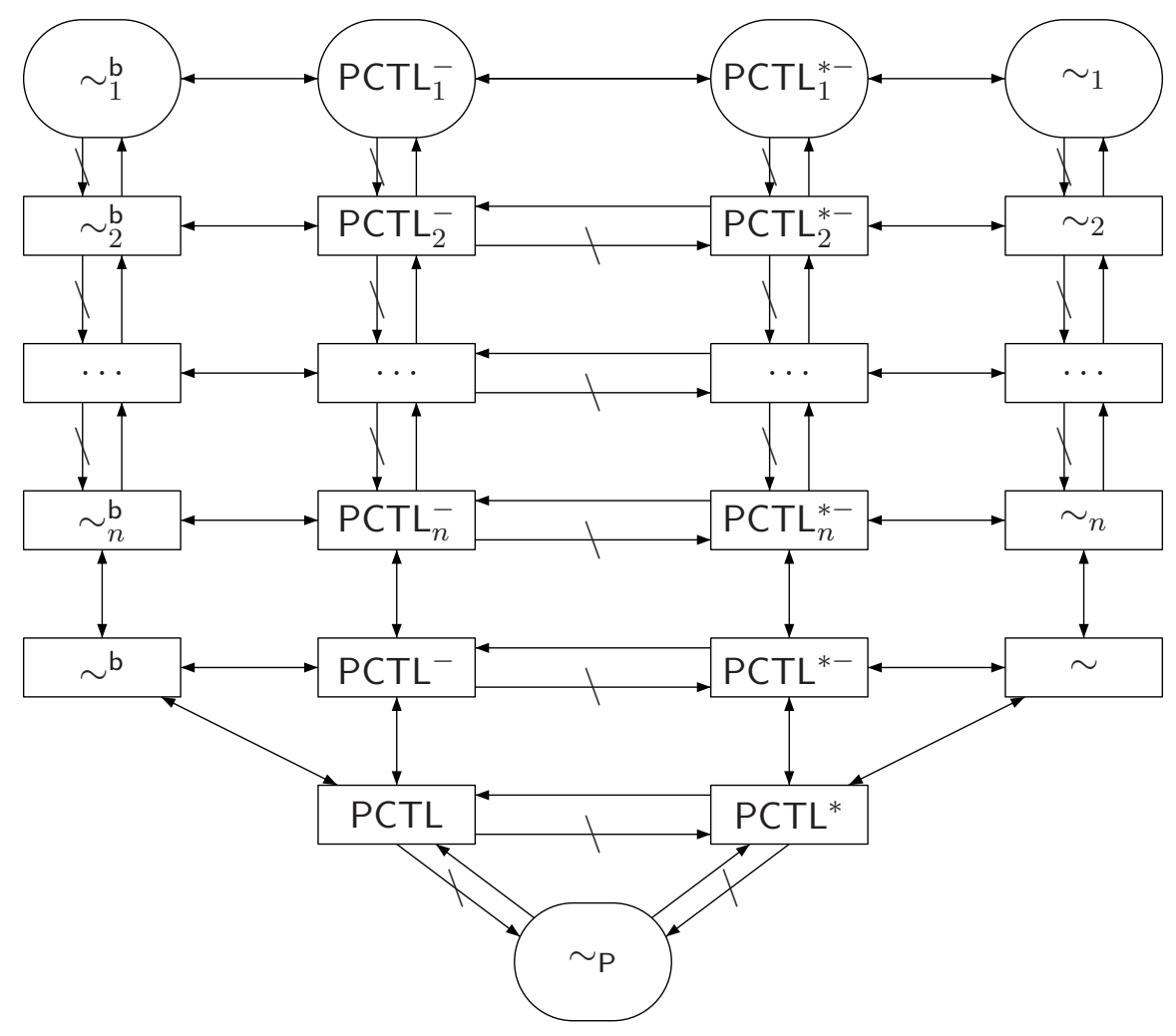

Figure 3: Relationship of different equivalences in strong scenario.

Example 5.1. Suppose $s$ and $r$ are given by Fig. 1 where each of $s_{1}$ and $s_{3}$ is attached with one transition respectively, that is, $s_{1} \rightarrow \mu_{1}$ such that $\mu_{1}\left(s_{4}\right)=0.4$ and $\mu_{1}\left(s_{5}\right)=0.6$, $s_{3} \rightarrow \mu_{3}$ such that $\mu_{3}\left(s_{4}\right)=0.4$ and $\mu_{3}\left(s_{5}\right)=0.6$. In addition, $s_{2}, s_{4}$ and $s_{5}$ only have a transition with probability 1 to themselves, and all these states are assumed to have different atomic propositions. Then $s \sim_{\mathrm{PCTL}} \mathrm{x}_{\mathrm{x}} r 1$ but $s \nsim_{\mathrm{PCTL}}{ }_{\backslash \mathrm{x}} r$, since we have a path formula

$$
\psi=\left(\left(L(s) \vee L\left(s_{1}\right)\right) \cup L\left(s_{5}\right)\right) \vee\left(\left(L(s) \vee L\left(s_{3}\right)\right) \cup L\left(s_{4}\right)\right)
$$

such that $s \models \mathcal{P}_{\leq 0.34}(\psi)$ but $r \not \forall \mathcal{P}_{\leq 0.34}(\psi)$, since there exists a scheduler $\sigma$ where the probability of paths satisfying $\psi$ in $\operatorname{Prob}_{\sigma, r}$ is equal to $\operatorname{Prob}_{\sigma, r}\left(C_{s s_{1} s_{5}}\right)+\operatorname{Prob}_{\sigma, r}\left(C_{s s_{3} s_{4}}\right)=$ 0.36. Note $\psi$ is not a $\mathrm{PCTL}_{\backslash \mathrm{x}}$ path formula.

In this section we shall introduce a notion of branching bisimulation. Similar to the definition of bounded reachability $\operatorname{Prob}_{\sigma, s}\left(C, C^{\prime}, n, \omega\right)$, we first define the function $\operatorname{Prob}_{\sigma, s}\left(C, C^{\prime}, \omega\right)$ which denotes the probability to go from $s$ to states in $C^{\prime}$ possibly via states in $C$. Again $\omega$ is used to keep track of the path which has been visited. Formally, $\operatorname{Prob}_{\sigma, s}\left(C, C^{\prime}, \omega\right)$ is equal to $\operatorname{Prob}_{\sigma, s}\left(C, C^{\prime}, n, \omega\right)$ when $n \rightarrow \infty$, i.e.,

$$
\operatorname{Prob}_{\sigma, s}\left(C, C^{\prime}, \omega\right)=\lim _{n \rightarrow \infty} \operatorname{Prob}_{\sigma, s}\left(C, C^{\prime}, n, \omega\right) .
$$

\footnotetext{
${ }^{1}$ This can be obtained by combining Theorem 5.6 and Example 5.5 in Section 5.2
} 
5.1. Weak probabilistic bisimulation. Before introducing our weak bisimulation, we give the classical definition of weak probabilistic bisimulation proposed in [35. Given an equivalence relation $\mathcal{R}, s$ can evolve into $\mu$ by a weak branching transition, written as $s \Rightarrow^{\mathcal{R}} \mu$, iff there exists a scheduler $\sigma$ such that $\mu(C)=\operatorname{Prob}_{\sigma, s}([s], C, s)$ for each $C \in S / \mathcal{R}$, where $[s]$ denotes the equivalence class containing $s$. Intuitively, $s \Rightarrow^{\mathcal{R}} \mu$ means that $s$ can evolve into $\mu$ only via states in $[s]$. Accordingly, weak branching combined transition $s \Rightarrow{ }_{\mathrm{P}}^{\mathcal{R}} \mu$ can be defined based on the weak branching transition, i.e. $s \Rightarrow_{\mathrm{P}}^{\mathcal{R}} \mu$ iff there exists a collection of weak branching transitions $\left\{s \Rightarrow^{\mathcal{R}} \mu_{i}\right\}_{i \in I}$, and a collection of probabilities $\left\{w_{i}\right\}_{i \in I}$ such that $\sum_{i \in I} w_{i}=1$ and $\mu=\sum_{i \in I} w_{i} \cdot \mu_{i}$.

We give the definition of weak probabilistic bisimulation as follows:

Definition 5.2. An equivalence relation $\mathcal{R} \subseteq S \times S$ is a weak probabilistic bisimulation iff $s \mathcal{R} r$ implies that $L(s)=L(r)$ and for each $s \rightarrow \mu$, there exists $r \Rightarrow_{\mathrm{P}}^{\mathcal{R}} \mu^{\prime}$ such that $\mu \mathcal{R} \mu^{\prime}$.

We write $s \simeq_{\mathrm{P}} r$ whenever there is a weak probabilistic bisimulation $\mathcal{R}$ such that $s \mathcal{R}$.

The following properties concerning weak probabilistic bisimulation are taken from [35]:

Lemma 5.3 ([35]).

(1) $\sim_{\mathrm{P}} \subset \sim_{\mathrm{PCTL}_{\backslash \mathrm{X}}^{*}} \subset \sim_{\mathrm{PCTL}} \mathrm{X}_{\mathrm{X}} \cdot$

$(2) \simeq_{\mathrm{P}}$ is preserved by $\|$.

5.2. A novel branching bisimulation. Below follows the definition of our branching bisimulation.

Definition 5.4. A relation $\mathcal{R} \subseteq S \times S$ is a branching bisimulation if $s \mathcal{R} r$ implies that $L(s)=L(r)$ and for any $\mathcal{R}$ downward closed sets $C, C^{\prime}$

(1) for each scheduler $\sigma$, there exists $\sigma^{\prime}$ such that $\operatorname{Prob}_{\sigma^{\prime}, r}\left(C, C^{\prime}, r\right) \geq \operatorname{Prob}_{\sigma, s}\left(C, C^{\prime}, s\right)$,

(2) for each scheduler $\sigma$, there exists $\sigma^{\prime}$ such that $\operatorname{Prob}_{\sigma^{\prime}, s}\left(C, C^{\prime}, s\right) \geq \operatorname{Prob}_{\sigma, r}\left(C, C^{\prime}, r\right)$.

We write $s \approx^{\mathrm{b}} r$ whenever there is a branching bisimulation $\mathcal{R}$ such that $s \mathcal{R} r$.

The following theorem shows that $\approx^{\mathrm{b}}$ is an equivalence relation. Also different from the strong cases where we use a series of equivalence relations to either characterize or approximate $\sim$ PCTL and $\sim_{\text {PCTL* }}$, in the weak scenario we show that $\approx^{b}$ itself is enough to characterize $\sim_{\mathrm{PCTL}} \mathrm{L}_{\mathrm{X}}$. Intuitively this is because in $\sim_{\mathrm{PCTL}} \mathrm{S}_{\backslash}$ only the unbounded until operator is allowed in path formulas which means we abstract from the number of steps to reach certain states.

Example 5.5. Refer to $s$ and $r$ described in Example 5.1, we can show that $\mathcal{R}=\{(s, r)\} \cup$ $I D$ is a branching bisimulation. The only non-trivial case is to show that $(s, r)$ satisfies the conditions of the relation. According to Definition 5.4. it is enough to check that for all the possible $C$ and $C^{\prime}$, the value of $\operatorname{Prob}_{\sigma_{m}, r}\left(C, C^{\prime}, r\right)$ will not be greater or smaller than $\operatorname{Prob}_{\sigma_{l}, s}\left(C, C^{\prime}, s\right)$ and $\operatorname{Prob}_{\sigma_{r}, s}\left(C, C^{\prime}, s\right)$ at the same time, where $\sigma_{m}$ is the scheduler of $r$ always choosing the middle transition, while $\sigma_{l}$ and $\sigma_{r}$ are schedulers of $s$ always choosing the left transition and the right transition of $s$ respectively.

Theorem 5.6.

$(1) \approx^{\mathrm{b}}$ is an equivalence relation.

$(2) \approx^{b}=\sim_{P C T L} \times$. 
Proof. The proof of the first clause is along the same line as the proof of Clause (1) of Lemma 4.6. For the second clause, let $\mathcal{R}=\left\{(s, r) \mid s \sim_{\mathrm{PCTL}} \times \mathrm{x}\right\}$ and $s \mathcal{R} r$, where $\mathcal{R}$ is obviously symmetric. We show that for any $\mathcal{R}$ closed sets $C, C^{\prime}$ and scheduler $\sigma$ of $s$, there exists a scheduler $\sigma^{\prime}$ of $r$ such that $\operatorname{Prob}_{\sigma^{\prime}, r}\left(C, C^{\prime}, r\right) \geq \operatorname{Prob}_{\sigma, s}\left(C, C^{\prime}, s\right)$. Following the way in the proof of Theorem 4.7, we can construct a formula $\varphi_{C}$ such that $\operatorname{Sat}\left(\varphi_{C}\right)=C$ where $C$ is a $\mathcal{R}$ closed set. Let $\psi=\varphi_{C} \bigcup \varphi_{C^{\prime}}$, then it is not hard to see that $s \models \neg \mathcal{P}_{<q}(\psi)$ where $q=\operatorname{Prob}_{\sigma, s}\left(C, C^{\prime}, s\right)$. By assumption $r \models \neg \mathcal{P}_{<q}(\psi)$, so there exists a scheduler $\sigma^{\prime}$ such that $\operatorname{Prob}_{\sigma^{\prime}, r}\left(C, C^{\prime}, r\right) \geq q$, that is, $\operatorname{Prob}_{\sigma^{\prime}, r}\left(C, C^{\prime}, r\right) \geq \operatorname{Prob}_{\sigma, s}\left(C, C^{\prime}, s\right)$.

The proof of $\approx^{\mathrm{b}} \subseteq \sim_{\mathrm{PCTL}}$ is by structural induction on the syntax of state formula $\varphi$ and path formula $\psi$ of $\mathrm{PCTL}_{\backslash \mathrm{x}}$, that is, we need to prove the following two results simultaneously.

(1) $s \approx^{\mathrm{b}} r$ implies that $s \models \varphi$ iff $r \models \varphi$ for any state formula $\varphi$.

(2) $\omega_{1} \approx^{\mathrm{b}} \omega_{2}$ implies that $\omega_{1} \models \psi$ iff $\omega_{2} \models \psi$ for any path formula $\psi$.

We only consider $\varphi=\mathcal{P}_{\leq q}(\psi)$ with $\psi=\varphi_{1} \cup \varphi_{2}$ since the other cases are similar. By induction hypothesis $\operatorname{Sat}\left(\varphi_{1}\right)$ and $\operatorname{Sat}\left(\varphi_{2}\right)$ are $\approx^{\mathrm{b}}$ closed, moreover $\operatorname{Prob}_{\sigma, s}(\{\omega \mid \omega \models$ $\psi\})=\operatorname{Prob}_{\sigma, s}\left(\operatorname{Sat}\left(\varphi_{1}\right), \operatorname{Sat}\left(\varphi_{2}\right), s\right)$ by Equation (5.1) for any $\sigma$. We prove by contradiction, and assume that $s \models \varphi$ and $r \not \models \varphi$. According to the semantics, $s \models \varphi$ iff $\forall \sigma \cdot \operatorname{Prob}_{\sigma, s}\left(\operatorname{Sat}\left(\varphi_{1}\right), \operatorname{Sat}\left(\varphi_{2}\right), s\right) \leq q$. If $r \not \forall \varphi$, then there exists $\sigma^{\prime}$ of $r$ such that it holds $\operatorname{Prob}_{\sigma^{\prime}, r}\left(\operatorname{Sat}\left(\varphi_{1}\right), \operatorname{Sat}\left(\varphi_{2}\right), r\right)>q$, therefore for such $\sigma^{\prime}$, there does not exist $\sigma$ of $s$ such that $\operatorname{Prob}_{\sigma, s}\left(\operatorname{Sat}\left(\varphi_{1}\right), \operatorname{Sat}\left(\varphi_{2}\right), s\right) \geq \operatorname{Prob}_{\sigma^{\prime}, r}\left(\operatorname{Sat}\left(\varphi_{1}\right), \operatorname{Sat}\left(\varphi_{2}\right), r\right)$, which contradicts the assumption $s \approx^{\mathrm{b}} r$. As a result, it must hold that $r \models \varphi$, and $s \sim_{\mathrm{PCTL}} \times \mathrm{x}$.

As in the strong scenario, $\approx^{\mathrm{b}}$ suffers from the same problem as $\sim_{i}^{\mathrm{b}}$ and $\sim_{i}$ with $i>1$, that is, it is not preserved by $\|$.

Counterexample 3. $s \approx^{\mathrm{b}} r$ does not necessarily imply $s\left\|t \approx^{\mathrm{b}} r\right\| t$ for any $t$. This can be shown in a similar way as Counterexample 1, since the result will still hold even if we replace the bounded until formula with an unbounded until formula in Counterexample 1 .

5.3. Weak bisimulations. In order to define weak bisimulation, we consider stuttering paths. Let $\Omega$ be a finite $\mathcal{R}$ downward closed path, then

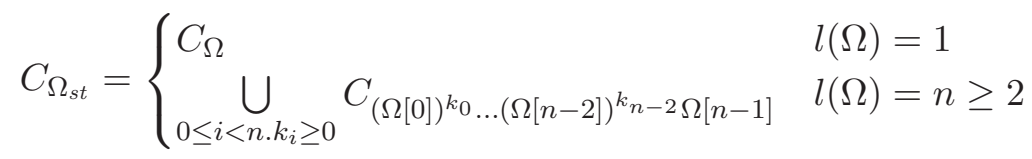

is the set of $\mathcal{R}$ downward closed paths which contain all stuttering paths, where $\Omega[i]$ denotes the $(i+1)$-th element in $\Omega$ such that $0 \leq i<l(\Omega)$. Accordingly, $C_{\widetilde{\Omega}_{s t}}=\underset{\Omega \in \widetilde{\Omega}}{\cup} C_{\Omega_{s t}}$ contains all the stuttering paths of each $\Omega \in \widetilde{\Omega}$.

Now we are ready to give the definition of weak bisimulation as follows:

Definition 5.7. A relation $\mathcal{R} \subseteq S \times S$ is a weak bisimulation if $s \mathcal{R} r$ implies that $L(s)=$ $L(r)$ and for any $\widetilde{\Omega} \subseteq\left(\mathcal{R}^{\downarrow}\right)^{+}$

(1) for each scheduler $\sigma$, there exists $\sigma^{\prime}$ such that $\operatorname{Prob}_{\sigma^{\prime}, r}\left(C_{\widetilde{\Omega}_{s t}}\right) \geq \operatorname{Prob}_{\sigma, s}\left(C_{\widetilde{\Omega}_{s t}}\right)$,

(2) for each scheduler $\sigma$, there exists $\sigma^{\prime}$ such that $\operatorname{Prob}_{\sigma^{\prime}, s}\left(C_{\widetilde{\Omega}_{s t}}\right) \geq \operatorname{Prob}_{\sigma, r}\left(C_{\widetilde{\Omega}_{s t}}\right)$.

We write $s \approx r$ whenever there is a weak bisimulation $\mathcal{R}$ such that $s \mathcal{R} r$. 
The following theorem shows that $\approx$ is an equivalence relation. For the same reason as in Theorem 5.6, $\approx$ is enough to characterize $\sim{ }_{\mathrm{PCTL}}{ }_{\mathrm{X}}^{*}$ which gives us the following theorem.

\section{Theorem 5.8 .}

$(1) \approx$ is an equivalence relation.

$(2) \approx=\sim_{\mathrm{PCTL}_{\backslash \mathrm{X}}^{*}}$.

Proof. The proof of the first clause is along the same line as the proof of Clause (1) of Lemma 4.6. For the second clause, let $\mathcal{R}=\left\{(s, r) \mid s \sim_{\mathrm{PCTL}_{\backslash \mathrm{x}}^{*}} r\right\}$ and $s \mathcal{R} r$, thus $\mathcal{R}$ is a symmetric relation. It suffices to show that for any $\widetilde{\Omega} \subseteq\left(\mathcal{R}^{\downarrow}\right)^{+}$and scheduler $\sigma$, there exists a scheduler $\sigma^{\prime}$ such that $\operatorname{Prob}_{\sigma^{\prime}, r}\left(C_{\widetilde{\Omega}_{s t}}\right) \geq \operatorname{Prob}_{\sigma, s}\left(C_{\widetilde{\Omega}_{s t}}\right)$. Following the way in the proof of Theorem 4.7. we can construct a formula $\varphi_{C}$ such that $\operatorname{Sat}\left(\varphi_{C}\right)=C$ where $C$ is a $\mathcal{R}$ closed set. Let $\psi_{\Omega}=\varphi_{C_{0}} \mathrm{U}\left(\varphi_{C_{1}} \mathrm{U} \ldots \varphi_{C_{n}}\right)$ where $\Omega=C_{C_{0} \ldots C_{n}}$, then $\psi_{\widetilde{\Omega}}=\underset{\Omega \in \widetilde{\Omega}}{\vee} \psi_{\Omega}$. It is easy to see that $s \models \neg \mathcal{P}_{<q}(\psi)$ where $q=\operatorname{Prob}_{\sigma, s}\left(C_{\widetilde{\Omega}_{s t}}\right)$ and $\psi=\psi_{\widetilde{\Omega}}$. By assumption $r \models \neg \mathcal{P}_{<q}(\psi)$, so there exists a scheduler $\sigma^{\prime}$ such that $\operatorname{Prob}_{\sigma^{\prime}, r}\left(C_{\widetilde{\Omega}_{s t}}\right) \geq q$, that is, $\operatorname{Prob}_{\sigma^{\prime}, r}\left(C_{\widetilde{\Omega}_{s t}}\right) \geq \operatorname{Prob}_{\sigma, s}\left(C_{\widetilde{\Omega}_{s t}}\right)$.

The proof of $\approx \subseteq \sim_{\mathrm{PCTL}}^{*}{ }_{\backslash \mathrm{X}}$ is by structural induction on the syntax of state formula $\varphi$ and path formula $\psi$ of $\mathrm{PCTL}_{\backslash \mathrm{X}}^{*}$, that is, we need to prove the following two results simultaneously.

(1) $s \approx r$ implies that $s \models \varphi$ iff $r \models \varphi$ for any state formula $\varphi$.

(2) $\omega_{1} \approx \omega_{2}$ implies that $\omega_{1} \models \psi$ iff $\omega_{2} \models \psi$ for any path formula $\psi$.

To make the proof clearer, we rewrite the syntax of $\mathrm{PCTL}_{\backslash \mathrm{X}}^{*}$ as follows which is equivalent to the original definition.

$$
\psi::=\varphi\left|\psi_{1} \vee \psi_{2}\right| \neg \psi \mid \psi_{1} \mathrm{U} \psi_{2}
$$

We only consider $\varphi^{\prime}=\mathcal{P}_{\leq q}(\psi)$ here. It suffices to prove that for each $\psi$, there exists $\widetilde{\Omega} \subseteq\left(\approx^{\downarrow}\right)^{+}$such that $C_{\widetilde{\Omega}}=\operatorname{Sat}(\psi)$. The proof is by structural induction on $\psi$ as follows:

(1) $\psi=\varphi$. By induction hypothesis $\operatorname{Sat}(\varphi)$ is $\approx \operatorname{closed}$. Let $\widetilde{\Omega}=\{\operatorname{Sat}(\varphi)\}$, then $C_{\widetilde{\Omega}}=$ $\operatorname{Sat}(\psi)$.

(2) $\psi=\psi_{1} \vee \psi_{2}$. By induction hypothesis there exist $\widetilde{\Omega}^{\prime}$ and $\widetilde{\Omega}^{\prime \prime}$ such that $\operatorname{Sat}\left(\psi_{1}\right)=C_{\widetilde{\Omega}_{s t}^{\prime}}$ and $\operatorname{Sat}\left(\psi_{2}\right)=C_{\widetilde{\Omega}_{s t}^{\prime \prime}}$. It is not hard to see that $\widetilde{\Omega}=\widetilde{\Omega}^{\prime} \cup \widetilde{\Omega}^{\prime \prime}$ will be enough.

(3) $\psi=\psi_{1} \cup \psi_{2}$. By induction hypothesis there exist $\widetilde{\Omega}^{\prime}$ and $\widetilde{\Omega}^{\prime \prime}$ such that $\operatorname{Sat}\left(\psi_{1}\right)=C_{\widetilde{\Omega}_{s t}^{\prime}}$ and $\operatorname{Sat}\left(\psi_{2}\right)=C_{\widetilde{\Omega}_{s t}^{\prime \prime}}$ Let $\widetilde{\Omega}=\widetilde{\Omega}^{\prime \prime} \cup\left\{\Omega^{\prime} \Omega^{\prime \prime} \mid \Omega^{\prime} \in \widetilde{\Omega}^{\prime} \wedge \Omega^{\prime \prime} \in \widetilde{\Omega}^{\prime \prime}\right\}$, then $C_{\widetilde{\Omega}}=\operatorname{Sat}(\psi)$.

(4) $\psi=\neg \psi^{\prime} . \quad s \models \mathcal{P}_{\geq q}(\psi)$ iff $s \models \mathcal{P}_{\leq 1-q}\left(\psi^{\prime}\right)$, so $\psi$ can be reduced to another formula without $\neg$ operator.

The remaining proof is routine and is omitted here.

Not surprisingly $\approx$ is not preserved by $\|$, which can be shown by using the same arguments as in Counterexample 3 ,

5.4. Taxonomy for weak bisimulation. As in the strong case, we summarize the equivalence relations in the weak scenario in Fig. 4, where all the denotations have the same meanings as Fig. 3. Compared to Fig. 3, Fig. 4 is much simpler because the step-indexed bisimulations are absent. As in the strong case, we do not consider the standard definition 


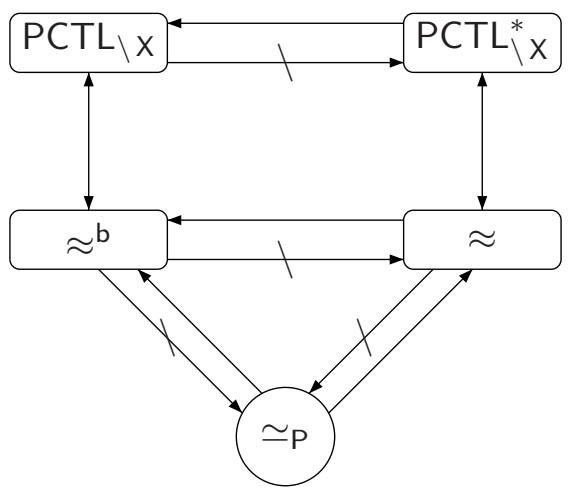

Figure 4: Relationship of different equivalences in weak scenario.

of weak bisimulation [35] which is a strict subset of $\simeq_{P}$ and can be defined by replacing $\Rightarrow{ }_{\mathrm{P}}^{\mathcal{R}}$ with $\Rightarrow^{\mathcal{R}}$ in Definition 5.2. Again, not surprisingly, all the relations shown in Fig. 4 coincide with the weak bisimulation defined in [5] over the DTMCs setting.

\section{Simulations}

In Section 4 and 5 we have discussed bisimulations and their characterizations. Two states $s$ and $r$ are bisimilar iff $s$ can mimic stepwise all the transitions of $r$ and vice versa. In this section we consider simulation relations that only require one direction mimicking. Simulations are preorders on the states, which have been used widely for verification purposes [27, 21, 19, 35, 5]. If $r$ simulates $s$, then $s$ can be seen as a correct implementation of $r$. Since $r$ is more abstract and contains less details, it is more preferable to be analysed, moreover some properties satisfied by $r$ are also preserved by $s$.

We shall discuss the characterization of simulations w.r.t. the safe fragments of PCTL and $\mathrm{PCTL}^{*}$. First let us introduce the safe fragment of $\mathrm{PCTL}^{*}$, denoted by $\mathrm{PCTL}_{\text {safe }}^{*}$, which is a fragment of $\mathrm{PCTL}^{*}$ without negative operators except for the atomic propositions, and is defined by the following syntax:

$$
\begin{aligned}
& \varphi::=a|\neg a| \varphi_{1} \wedge \varphi_{2}\left|\varphi_{1} \vee \varphi_{2}\right| \mathcal{P}_{\leq q}(\psi) \\
& \psi::=\varphi\left|\psi_{1} \wedge \psi_{2}\right| \psi_{1} \vee \psi_{2}|\mathrm{X} \psi| \psi_{1} \bigcup \psi_{2}
\end{aligned}
$$

where $a \in A P$ and $q \in[0,1]$. Accordingly, the safe fragment of PCTL, denoted by $\mathrm{PCTL}_{\text {safe }}$, is a sub logic of PCTL safe where the path formulas are constrained to be the following form:

$$
\psi::=\mathrm{X} \varphi\left|\varphi_{1} \mathrm{U} \varphi_{2}\right| \varphi_{1} \mathrm{U}^{\leq n} \varphi_{2} .
$$

We write $s \prec_{\mathrm{PCTL}_{\text {safe }}^{*}}^{*} r$ iff $r \models \varphi$ implies that $s \models \varphi$ for any $\varphi$ of $\mathrm{PCTL}_{\text {safe }}^{*}$, and similarly for other sub-logics.

Below we recall the notion of weight functions [22, and then use them to define strong probabilistic simulation relations [35]:

Definition 6.1. Let $\mathcal{R}=S \times S$ be a relation over $S$. A weight function for $\mu$ and $\nu$ with respect to $\mathcal{R}$ is a function $\Delta: S \times S \mapsto[0,1]$ such that:

- $\Delta(s, r)>0$ implies that $s \mathcal{R} r$,

- $\mu(s)=\sum_{r \in S} \Delta(s, r)$ for any $s \in S$,

- $\nu(r)=\sum_{s \in S} \Delta(s, r)$ for any $r \in S$. 
We write $\mu \sqsubseteq_{\mathcal{R}} \nu$ iff there exists a weight function for $\mu$ and $\nu$ with respect to $\mathcal{R}$.

Definition 6.2. A relation $\mathcal{R} \subseteq S \times S$ is a strong probabilistic simulation iff $s \mathcal{R} r$ implies that $L(s)=L(r)$ and for each $s \rightarrow \mu$, there exists a combined transition $r \rightarrow_{\mathrm{P}} \mu^{\prime}$ such that $\mu \sqsubseteq \mathcal{R} \mu^{\prime}$.

We write $s \prec_{\mathrm{P}} \quad r$ whenever there is a strong probabilistic simulation $\mathcal{R}$ such that $s \mathcal{R} r$.

It was shown in [35] that $\sqsubseteq_{\mathcal{R}}$ is a congruence, i.e. $s \prec_{\mathrm{P}} r$ implies that $s\left\|t \prec_{\mathrm{p}} r\right\| t$ for any $t$. But not surprisingly, it turns out that the strong probabilistic simulation is too fine w.r.t $\prec \mathrm{PCTL}_{\text {safe }}$ and $\prec \mathrm{PCTL}_{\text {safe }}^{*}$ which can be seen from Example 3.4. Similarly we have an analogue of Theorem 3.6 in the simulation scenario, where we only consider the safe fragment of the logics, thus the subscription safe is often omitted for readability.

\section{Theorem 6.3.}

(1) $\prec_{\mathrm{PCTL}}, \prec_{\mathrm{PCTL}^{*}}, \prec_{\mathrm{PCTL}^{-}}, \prec_{\mathrm{PCTL}_{i}^{-}}, \prec_{\mathrm{PCTL}^{*-}}, \prec_{\mathrm{PCTL}_{i}^{*-}}$, and $\prec_{\mathrm{P}}$ are preorders for any $i \geq 1$.

(2) $\prec_{\mathrm{P}} \subset \prec_{\mathrm{PCTL}^{*}} \subset \prec_{\mathrm{PCTL}}$.

(3) $\prec_{\mathrm{PCTL}^{*-}} \subset \prec_{\mathrm{PCTL}^{-}}$

(4) $\prec_{\mathrm{PCTL}_{1}^{*-}}=\prec_{\mathrm{PCTL}_{1}^{-}}$.

(5) $\prec_{\mathrm{PCTL}_{i}^{*-}} \subset \prec_{\mathrm{PCTL}_{i}^{-}}$for any $i>1$.

(6) $\prec \mathrm{PCTL} \subset \prec_{\mathrm{PCTL}^{-}} \subset \prec_{\mathrm{PCTL}_{i+1}^{-}} \subset \prec_{\mathrm{PCTL}_{i}^{-}}$for all $i \geq 0$.

(7) $\prec_{\mathrm{PCTL}^{*}} \subset \prec_{\mathrm{PCTL}^{*-}} \subset \prec_{\mathrm{PCTL}_{i+1}^{*-}} \subset \prec_{\mathrm{PCTL}_{i}^{*-}}$ for all $i \geq 0$.

Proof. For Clause (1) we only prove that $\prec_{\mathrm{PCTL}}$ is a preorder since the others are similar. The reflexivity is trivial as $s \prec_{\mathrm{PCTL}} s$ for any $s$. Suppose that $s \prec_{\mathrm{PCTL}} t$ and $t \prec_{\mathrm{PCTL}} r$, then we need to prove that $s \prec$ PCTL $r$ in order to show the transitivity. According to the definition of $\prec_{\mathrm{PCTL}}$, we need to prove that $r \models \varphi$ implies $s \models \varphi$ for any $\varphi$. Suppose that $r \models \varphi$ for some $\varphi$, then $t \models \varphi$ because of $t \prec$ PCTL $r$, moreover since $s \prec$ PCTL $t$, hence $s \models \varphi$ which completes the proof.

The proof of Clause (2) can be found in 35 .

In order to prove Clause (4), we can follow the same reasoning as in Theorem 3.6, by showing that the safe fragments of $\mathrm{PCTL}_{1}^{-}$and $\mathrm{PCTL}_{1}^{*-}$ coincide. Again the syntax of path formulas of safe $\mathrm{PCTL}_{1}^{*-}$ can be rewritten as:

$$
\psi::=\varphi|\mathbf{X} \varphi| \psi_{1} \wedge \psi_{2} \mid \psi_{1} \vee \psi_{2},
$$

therefore only the following cases need to be considered: $\mathcal{P}_{\leq q}(\varphi), \mathcal{P}_{\leq q}\left(\mathbf{X} \varphi_{1} \wedge \mathbf{X} \varphi_{2}\right), \mathcal{P}_{\leq q}\left(\mathbf{X} \varphi_{1} \wedge\right.$ $\left.\varphi_{2}\right), \mathcal{P}_{\leq q}\left(\mathbf{X} \varphi_{1} \vee \mathbf{X} \varphi_{2}\right), \mathcal{P}_{\leq q}\left(\mathbf{X} \varphi_{1} \vee \varphi_{2}\right)$, all of which can be transformed to a formula in safe $\mathrm{PCTL}_{1}^{-}$. For instance $s \models \mathcal{P}_{\leq q}\left(\mathbf{X} \varphi_{1} \vee \varphi_{2}\right)$ iff $s \models \varphi_{2} \vee s \models \mathcal{P}_{\leq q}\left(\mathbf{X} \varphi_{1}\right)$. The remaining proof is similar and omitted here.

The proofs of all the other clauses are trivial.

6.1. Strong $i$-depth branching simulation. Following Section 4.2 we can define strong $i$-depth branching simulation which can be characterized by $\prec_{\mathrm{PCTL}_{i}^{-}}$. Let $s \quad \prec_{0}^{\mathrm{b}} r$ iff $L(s)=L(r)$, then

Definition 6.4. A relation $\mathcal{R} \subseteq S \times S$ is a strong $i$-depth branching simulation with $i \geq 1$ iff $s \mathcal{R} r$ implies that $s \prec_{i-1}^{\mathrm{b}} r$ and for any $\mathcal{R}$ downward closed sets $C, C^{\prime}$, and any scheduler $\sigma$, there exists $\sigma^{\prime}$ such that $\operatorname{Prob}_{\sigma^{\prime}, r}\left(C, C^{\prime}, i, r\right) \geq \operatorname{Prob}_{\sigma, s}\left(C, C^{\prime}, i, s\right)$. 
We write $s \prec_{i}^{\mathrm{b}} r$ whenever there is a strong $i$-depth branching simulation $\mathcal{R}$ such that $s \mathcal{R} r$. The strong branching simulation $\prec^{\mathrm{b}}$ is defined as $\prec^{\mathrm{b}}=\cap_{i \geq 0} \prec_{i}^{\mathrm{b}}$.

Below we show properties similar to Lemma 4.6 for strong $i$-depth branching simulation.

\section{Lemma 6.5.}

(1) $\prec^{\mathrm{b}}$ and $\prec_{i}^{\mathrm{b}}$ are preorders for any $i \geq 0$.

(2) $\prec_{j}^{\mathrm{b}} \subseteq \prec_{i}^{\mathrm{b}}$ provided that $0 \leq i \leq j$.

(3) There exists $i \geq 0$ such that $\prec_{j}^{\mathrm{b}}=\prec_{k}^{\mathrm{b}}$ for any $j, k \geq i$.

Proof. We consider the first clause. The reflexivity is trivial, we only prove the transitivity. Suppose that $s_{1} \quad \prec_{i}^{\mathrm{b}} \quad s_{2}$ and $s_{2} \quad \prec_{i}^{\mathrm{b}} s_{3}$, we need to prove that $s_{1} \quad \prec_{i}^{\mathrm{b}} s_{3}$. By Definition 6.4 there exists strong simulation $\mathcal{R}_{1}$ and $\mathcal{R}_{2}$ such that $s_{1} \mathcal{R}_{1} s_{2}$ and $s_{2} \mathcal{R}_{2} s_{3}$. Moreover since $s \quad \prec_{i}^{\mathrm{b}} s$ for any $s$, reflexive relations $\mathcal{R}_{1}$ and $\mathcal{R}_{2}$ always exist. Let $\mathcal{R}=\mathcal{R}_{1} \circ \mathcal{R}_{2}=\left\{\left(s_{1}, s_{3}\right) \mid \exists s_{2} .\left(s_{1} \mathcal{R}_{1} s_{2} \wedge s_{2} \mathcal{R}_{2} s_{3}\right)\right\}$, it is enough to prove that $\mathcal{R}$ is strong $i$-depth branching simulation. Due to the reflexivity, any $\mathcal{R}$ downward closed set $C$ is also $\mathcal{R}_{1}$ and $\mathcal{R}_{2}$ downward closed. Therefore for any $\mathcal{R}$ downward closed sets $C, C^{\prime}$ and a scheduler $\sigma$, there exists $\sigma^{\prime}$ such that $\operatorname{Prob}_{\sigma^{\prime}, s_{2}}\left(C, C^{\prime}, i, s_{2}\right) \geq \operatorname{Prob}_{\sigma, s_{1}}\left(C, C^{\prime}, i, s_{1}\right)$ according to Definition 6.4. Similarly, there exists $\sigma^{\prime \prime}$ such that $\operatorname{Prob}_{\sigma^{\prime \prime}, s_{3}}\left(C, C^{\prime}, i, s_{3}\right) \geq$ $\operatorname{Prob}_{\sigma^{\prime}, s_{2}}\left(C, C^{\prime}, i, s_{2}\right) \geq \operatorname{Prob}_{\sigma, s_{1}}\left(C, C^{\prime}, i, s_{1}\right)$, and $\mathcal{R}$ is indeed a strong $i$-depth branching simulation. This completes the proof.

The second clause follows directly from Definition 6.4.

For the third clause, note there are only finitely many states, thus in the worst case each state is only able to simulate itself, and there always exists $i$ such that $\sim_{j}$ is stable for all $j \geq i$.

Our strong $i$-depth branching simulation coincides with $\prec_{\mathrm{PCTL}_{i}^{-}}$for each $i$, therefore $\prec$ PCTL is equivalent to $\prec^{\mathrm{b}}$ as shown by the following theorem.

Theorem 6.6. $\prec_{\mathrm{PCTL}_{i}^{-}}=\prec_{i}^{\mathrm{b}}$ for any $i \geq 1$, and moreover $\prec_{\mathrm{PCTL}}=\prec^{\mathrm{b}}$.

Proof. We first prove that $\prec_{\mathrm{PCTL}_{i}^{-}}$implies $\prec_{i}^{\mathrm{b}}$. Let $\mathcal{R}=\left\{(s, r) \mid s \prec_{\mathrm{PCTL}_{i}^{-}} r\right\}$ and $s \mathcal{R} r$, we need to prove that for any $\mathcal{R}$ downward closed sets $C, C^{\prime}$ and scheduler $\sigma$ of $s$, there exists $\sigma^{\prime}$ of $r$ such that $\operatorname{Prob}_{\sigma^{\prime}, r}\left(C, C^{\prime}, i, r\right) \geq \operatorname{Prob}_{\sigma, s}\left(C, C^{\prime}, i, s\right)$. Note that $\operatorname{Sat}(\varphi)$ is a $\mathcal{R}$ downward closed set for any $\varphi$. Since the states space is finite, for each $\mathcal{R}$ downward closed set $C$, there exists $\varphi_{C}$ such that $\operatorname{Sat}\left(\varphi_{C}\right)=C$. Assume that there exists $\mathcal{R}$ downward closed sets $C, C^{\prime}$ and $\sigma$ such that $\operatorname{Prob}_{\sigma^{\prime}, r}\left(C, C^{\prime}, i, r\right)<\operatorname{Prob}_{\sigma, s}\left(C, C^{\prime}, i, s\right)$ for all schedulers $\sigma^{\prime}$ of $r$. Then there exists $\operatorname{Prob}_{\sigma^{\prime}, r}\left(C, C^{\prime}, i, r\right) \leq q<\operatorname{Prob}_{\sigma, s}\left(C, C^{\prime}, i, s\right)$ such that $r \models \mathcal{P}_{\leq q}(\psi)$ but $s \not \forall \mathcal{P}_{\leq q}(\psi)$ where $\psi=\varphi_{C} \mathrm{U} \leq i \varphi_{C^{\prime}}$, this contradicts the assumption that $s \prec_{\mathrm{PCTL}_{i}^{-}} r$. Therefore $\mathcal{R}$ is a strong $i$-depth branching simulation.

In order to prove that $\prec_{i}^{\mathrm{b}}$ implies $\prec_{\mathrm{PCTL}_{i}^{-}}$, we need to prove that whenever $s \prec_{i}^{\mathrm{b}} r$ and $r \models \varphi$, we also have $s \models \varphi$. We prove by structural induction on $\varphi$, and only consider the case when $\varphi=\mathcal{P}_{\leq q}\left(\varphi_{1} \mathrm{U}^{\leq i} \varphi_{2}\right)$ since all the others are trivial. By induction hypothesis $\operatorname{Sat}\left(\varphi_{1}\right)$ and $\operatorname{Sat}\left(\varphi_{2}\right)$ are $\prec_{i}^{\mathrm{b}}$ downward closed, therefore if $r \models \mathcal{P}_{\leq q}\left(\varphi_{1} \mathrm{U}^{\leq i} \varphi_{2}\right)$, but $s \not$ $\mathcal{P}_{\leq q}\left(\varphi_{1} \mathrm{U}^{\leq i} \varphi_{2}\right)$, then there exists a scheduler $\sigma$ of $s$ such that there does not exist $\sigma^{\prime}$ such that $\operatorname{Prob}_{\sigma^{\prime}, r}\left(\operatorname{Sat}\left(\varphi_{1}\right), \operatorname{Sat}\left(\varphi_{2}\right), i, r\right) \geq \operatorname{Prob}_{\sigma, s}\left(\operatorname{Sat}\left(\varphi_{1}\right), \operatorname{Sat}\left(\varphi_{2}\right), i, s\right)$ which contradicts the assumption that $s \prec_{i}^{\mathrm{b}} r$. 

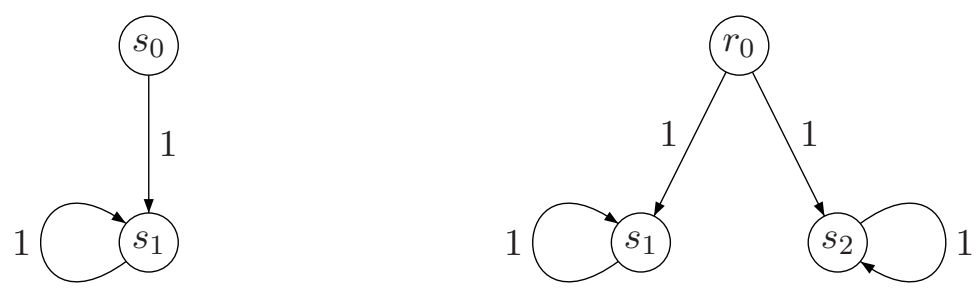

Figure 5: An example illustrating $s_{0} \nprec \mathrm{PCTL}_{\text {live }} r_{0}$.

In Counterexample 1 we have shown the $\sim_{i}^{\mathrm{b}}$ is not compositional for $i>1$, using the same arguments we can show that $\prec_{i}^{\mathrm{b}}$ is not compositional either for $i>1$, thus we have:

Lemma 6.7. $s \quad \prec_{1}^{\mathrm{b}} r$ implies that $s\left\|t \quad \prec_{1}^{\mathrm{b}} r\right\| t$ for any $t$, while $\prec_{i}^{\mathrm{b}}$ with $i>1$ is not compositional in general.

Proof. Let $\mathcal{R}=\left\{(s\|t, r\| t) \mid s \prec_{1}^{\mathrm{b}} r\right\}$, it is enough to show that $\mathcal{R}$ is a strong 1-depth simulation. Let $C^{\prime}$ be a $\prec_{1}^{\mathrm{b}}$ downward closed set, then $\left\{s^{\prime} \| t \mid s^{\prime} \in C^{\prime}\right\}$ is $\mathcal{R}$ downward closed, the following proof is similar with the proof of Lemma 4.4,

Note that Counterexample 1 also applies here, thus $\prec_{i}^{\mathrm{b}}$ is not necessarily compositional when $i>1$.

Remark 6.8. The safe fragment of PCTL we adopt in this paper is slightly different from 5] where two new operators $\widetilde{X}$ and $\widetilde{U}$ are introduced, called weak next and until respectively, and $\mathcal{P}_{\leq q}(\psi)$ is replaced by $\mathcal{P}_{\geq q}(\psi)$. The semantics of $\widetilde{\mathbf{X}}$ and $\widetilde{U}$ are defined as follows where $|\omega|$ denotes the length of $\omega$ :

$$
\begin{gathered}
\omega \models \widetilde{\mathrm{X}} \varphi \text { iff }(|\omega|<1 \vee \omega[i] \models \varphi) \\
\omega \models \varphi_{1} \widetilde{\mathrm{U}} \varphi_{2} \text { iff }\left(\omega \models \varphi_{1} \cup \varphi_{2} \vee \forall i \leq|\omega| . \omega[i] \models \varphi_{1}\right)
\end{gathered}
$$

Similarly we can also define the weak counterpart of the bounded until $\widetilde{U}^{\leq n}$. Due to the duality between $\mathbf{X}, \mathbf{U}^{\leq n}, \mathbf{U}$ and their weak counterparts, these two variants of safe PCTL are essentially equivalent, and we refer to $[5]$ for detailed discussions.

Let $\mathrm{PCTL}_{\text {live }}$ denote the liveness fragment of PCTL in [5] which is the same as $\mathrm{PCTL}_{\text {safe }}$

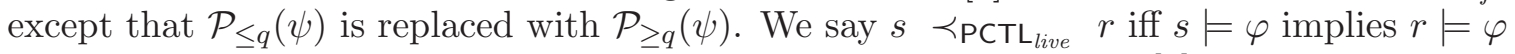
for any state formula of $\mathrm{PCTL}$ live. Even though it has been shown in [5] that $\prec \mathrm{PCTL}_{\text {safe }}$ and $\prec \mathrm{PCTL}_{\text {live }}$ are equivalent for DTMCs, the result is not true for PAs. Refer to the following example.

Example 6.9. Consider the two states $s_{0}$ and $r_{0}$ shown in Fig. 5 , where we assume that all the states have different labels except that $L\left(s_{0}\right)=L\left(r_{0}\right)$. It is easy to check that $s_{0} \prec_{\mathrm{P}} r_{0}$, thus $s_{0} \prec \mathrm{PCTL}_{\text {safe }} r_{0}$ according to Clause (2) of Theorem 6.3, but we have $s_{0} \nprec_{\mathrm{PCTL}} \mathrm{L}_{\text {live }} r_{0}$. Let $\varphi=\mathcal{P}_{\geq 1}\left(L\left(s_{0}\right) \cup L\left(s_{1}\right)\right)$ which is a valid state formula of $\mathrm{PCTL}_{\text {live }}$, it is obvious that $s_{0} \models \varphi$, but $r_{0} \not \models \varphi$ since the minimal probability of $r_{0}$ reaching state $s_{1}$ is equal to 0 i.e. by choosing the transition to $s_{2}$.

6.2. Strong $i$-depth simulation. In this section we introduce strong $i$-depth simulation which can be characterized by $\prec_{\mathrm{PCTL}_{i}^{*-}}$. Below follows the definition of strong $i$-depth simulation where $\prec_{0}=\prec_{0}^{b}$. 
Definition 6.10. A relation $\mathcal{R} \subseteq S \times S$ is a strong $i$-depth simulation with $i \geq 1$ iff $s \mathcal{R} r$ implies that $s \prec_{i-1} \quad r$ and for any $\widetilde{\Omega} \subseteq\left(\mathcal{R}^{\downarrow}\right)^{+}$with $l(\widetilde{\Omega})=i$ and any scheduler $\sigma$, there exists $\sigma^{\prime}$ such that $\operatorname{Prob}_{\sigma^{\prime}, r}\left(C_{\widetilde{\Omega}}\right) \geq \operatorname{Prob}_{\sigma, s}\left(C_{\widetilde{\Omega}}\right)$.

We write $s \prec_{i} \quad r$ whenever there is a strong $i$-depth simulation $\mathcal{R}$ such that $s \mathcal{R} r$. The strong simulation $\prec$ is defined as $\prec=\cap_{i \geq 0} \prec_{i}$.

Below we show properties similar to Lemma 4.9 for strong $i$-depth simulations.

\section{Lemma 6.11.}

(1) $\prec$ and $\prec_{i}$ are preorders for any $i \geq 0$.

(2) $\prec_{j} \subseteq \prec_{i}$ provided that $0 \leq i \leq j$.

(3) There exists $i \geq 0$ such that $\prec_{j}=\prec_{k}$ for any $j, k \geq i$.

Proof.

(1) This clause can be proved in a similar way as Clause (1) of Lemma 6.5.

(2) According to Definition 6.10, as $i$ is increasing, $\prec_{i}$ is getting finer.

(3) The proof is based on the fact that the number of states is finite, with the similar argument as in Clause (3) of Lemma 6.5.

Our strong $i$-depth simulation coincides with $\prec_{\mathrm{PCTL}_{i}^{*-}}$ for each $i$, therefore $\prec_{\mathrm{PCTL}^{*}}$ is equivalent to $\prec$ as shown by the following theorem.

Theorem 6.12. $\prec_{\mathrm{PCTL}_{i}^{*-}}=\prec_{i}$ for any $i \geq 1$, and moreover $\prec_{\mathrm{PCTL}}{ }^{*}=\prec$.

Proof. We first prove that $s \prec_{\mathrm{PCTL}_{i}^{*-}} \quad r$ implies $s \quad \prec_{i} \quad r$ for any $s$ and $r$. Let $\mathcal{R}=$ $\left\{(s, r) \mid s \prec_{\mathrm{PCTL}_{i}^{*-}} r\right\}$ and $s \mathcal{R} r$, we need to show that for any $\widetilde{\Omega} \subseteq\left(\mathcal{R}^{\downarrow}\right)^{+}$with $l(\widetilde{\Omega}) \leq i$ and scheduler $\sigma$, there exists a scheduler $\sigma^{\prime}$ such that $\operatorname{Prob}_{\sigma^{\prime}, r}\left(C_{\widetilde{\Omega}}\right) \geq \operatorname{Prob}_{\sigma, s}\left(C_{\widetilde{\Omega}}\right)$. By the definition of $\mathcal{R}$, there exists a formula $\varphi_{C}$ such that $\operatorname{Sat}\left(\varphi_{C}\right)=C$ where $C$ is an $\mathcal{R}$ downward closed set. Suppose $\Omega=C_{0} C_{1} \ldots C_{j}$ with $j \leq i$, then

$$
\psi_{\Omega}=\varphi_{C_{0}} \wedge \mathrm{X}\left(\varphi_{C_{1}} \wedge \ldots \wedge \mathrm{X}\left(\varphi_{C_{j-1}} \wedge \mathrm{X} \varphi_{C_{j}}\right) \ldots\right)
$$

can be used to characterize $\Omega$, that is, $\operatorname{Sat}\left(\psi_{\Omega}\right)=C_{\Omega}$. Let $\psi=\underset{\Omega \in \widetilde{\Omega}}{\vee} \psi_{\Omega}$, then $\operatorname{Sat}(\psi)=C_{\widetilde{\Omega}}$. We proceed by contradiction. Suppose that there does not exist $\sigma^{\prime}$ such that $\operatorname{Prob}_{\sigma^{\prime}, r}\left(C_{\widetilde{\Omega}}\right) \geq$ $\operatorname{Prob}_{\sigma, s}\left(C_{\widetilde{\Omega}}\right)$, then there exists $q$ such that $r \models \mathcal{P}_{\leq q}(\psi)$, but $s \not \models \mathcal{P}_{\leq q}(\psi)$ which contradicts the assumption that $s \prec_{\mathrm{PCTL}_{i}^{*-}} r$, so there exists a scheduler $\sigma^{\prime}$ such that $\operatorname{Prob}_{\sigma^{\prime}, r}\left(C_{\widetilde{\Omega}}\right) \geq$ $q=\operatorname{Prob}_{\sigma, s}\left(C_{\widetilde{\Omega}}\right)$.

The proof of $\prec_{i} \subseteq \prec_{\mathrm{PCTL}_{i}^{*-}}$ is by structural induction on the syntax of state formula $\varphi$ and path formula $\psi$ of safe $\mathrm{PCTL}_{i}^{*-}$, that is, we need to prove the following two results simultaneously.

(1) $r \models \varphi$ implies $s=\varphi$ for any state formula $\varphi$ provided that $s \prec_{i} r$.

(2) $\omega_{2} \models \psi$ implies $\omega_{1} \models \psi$ for any path formula $\psi$ provided that $\omega_{1} \prec_{i} \omega_{2}$.

We only consider $\varphi=\mathcal{P}_{\leq q}(\psi)$ such that $\operatorname{Depth}(\psi) \leq i$ here. Suppose that $r \models \varphi$, i.e. $\forall \sigma \cdot \operatorname{Prob}_{\sigma, r}(\{\omega \mid \omega \models \psi\}) \leq q$, we need to show that $s \models \varphi$. We proceed by contradiction, and assume that $s \not \models \varphi$, i.e. there exists $\sigma$ such that $\operatorname{Prob}_{\sigma, s}(\{\omega \mid \omega \models \psi\})>q$. By induction hypothesis $\{\omega \mid \omega \models \psi\}$ is $\prec_{i}$ downward closed. Since Depth $(\psi) \leq i$, there exists $\widetilde{\Omega}$ such that $l(\widetilde{\Omega}) \leq i$ and $C_{\widetilde{\Omega}}=\{\omega \mid \omega \models \psi\}$. Since $r \models \varphi$, there does not exists $\sigma^{\prime}$ such that $\operatorname{Prob}_{\sigma^{\prime}, r}\left(C_{\widetilde{\Omega}}\right) \geq \operatorname{Prob}_{\sigma, s}\left(C_{\widetilde{\Omega}}\right)=q$, which contradicts the assumption that $s \prec_{i} r$, thus it holds that $s \models \varphi$. 
Similarly, we can show that $\prec_{i}$ is not compositional either for $i>1$, thus we have:

Lemma 6.13. $s \prec_{1} r$ implies that $s\left\|t \prec_{1} r\right\| t$ for any $t$, while $\prec_{i}$ with $i>1$ is not compositional in general.

Proof. According to Theorem 6.6 and 6.12, and Clause (4) of Theorem 6.3, $\prec_{1}^{\mathrm{b}}=\prec_{1}$, thus the result is straightforward according to Lemma 6.7

6.3. Weak simulation. Given the results for weak bisimulation from Section [5, the characterization of weak simulation is straightforward. Let us first introduce the definition of weak probabilistic simulation by Segala and Lynch [35] as follows:

Definition 6.14. A relation $\mathcal{R} \subseteq S \times S$ is a weak probabilistic simulation iff $s \mathcal{R} r$ implies that $L(s)=L(r)$ and for each $s \rightarrow \mu$, there exists $r \Rightarrow{ }_{\mathrm{P}}^{\mathcal{R}} \mu^{\prime}$ such that $\mu \sqsubseteq_{\mathcal{R}} \mu^{\prime}$.

We write $s \precsim \mathrm{P} r$ whenever there is a weak probabilistic simulation $\mathcal{R}$ such that $s \mathcal{R} r$. From [35] we know that $\precsim \mathrm{P}$ is compositional, but it is too fine for $\precsim \mathrm{PCTL}_{\backslash \mathrm{x}}$ as well as $\precsim \mathrm{PCTL}_{\perp x}^{*}$, therefore along the line of weak bisimulation, we have similar results for weak simulation. Below follows the definition of branching simulation.

Definition 6.15. A relation $\mathcal{R} \subseteq S \times S$ is a branching simulation iff $s \mathcal{R} r$ implies that $L(s)=L(r)$ and for any $\mathcal{R}$ downward closed sets $C, C^{\prime}$ and any scheduler $\sigma$, there exists $\sigma^{\prime}$ such that $\operatorname{Prob}_{\sigma^{\prime}, r}\left(C, C^{\prime}, r\right) \geq \operatorname{Prob}_{\sigma, s}\left(C, C^{\prime}, s\right)$.

We write $s \precsim r$ whenever there is a branching simulation $\mathcal{R}$ such that $s \mathcal{R} r$.

Due to Counterexample 3 , $\precsim^{\mathrm{b}}$ is not compositional, but it coincides with $\precsim \mathrm{PCTL}_{\backslash \mathrm{X}}$ as shown by the following theorem.

Theorem 6.16. $\precsim^{\mathrm{b}}$ is a preorder, and $\precsim^{\mathrm{b}}=\precsim_{\mathrm{PCTL}} \mathrm{x}$.

Proof. The proof of the first statement is along the same line as the proof of Clause (1) of Lemma 6.5. For the second statement, we prove that $s \underset{\approx}{ }$ PCTL $\backslash$ x $r$ implies $s \underset{\approx}{ } r$ for any $s$ and $r$. Let $\mathcal{R}=\left\{(s, r) \mid s \underset{\precsim \mathrm{PCTL}_{\perp \mathrm{x}}}{r}\right\}$ and $s \mathcal{R} \underset{r}{ }$, we need to prove that for any $\mathcal{R}$ downward closed sets $C, C^{\prime}$ and scheduler $\sigma$, there exists a scheduler $\sigma^{\prime}$ such that $\operatorname{Prob}_{\sigma^{\prime}, r}\left(C, C^{\prime}, r\right) \geq \operatorname{Prob}_{\sigma, s}\left(C, C^{\prime}, s\right)$. Let $\varphi_{C}$ be a formula such that $\operatorname{Sat}\left(\varphi_{C}\right)=C$ where $C$ is a $\mathcal{R}$ downward closed set. We proceed by contradiction. Suppose that there does not exist $\sigma^{\prime}$ such that $\operatorname{Prob}_{\sigma^{\prime}, r}\left(C, C^{\prime}, r\right) \geq \operatorname{Prob}_{\sigma, s}\left(C, C^{\prime}, s\right)$, then there exists $q$ such that $r \models \mathcal{P}_{\leq q}(\psi)$ where $\psi=\varphi_{C} \bigcup \varphi_{C^{\prime}}$, but $s \not \forall \mathcal{P}_{\leq q}(\psi)$, which contradicts the assumption that $s \underset{\precsim \mathrm{PCTL}_{\backslash \mathrm{x}}}{ } r$. Therefore there must exist a scheduler $\sigma^{\prime}$ such that $\operatorname{Prob}_{\sigma^{\prime}, r}\left(C, C^{\prime}, r\right) \geq \operatorname{Prob}_{\sigma, s}\left(C, C^{\prime}, s\right)$.

The proof of $\precsim \mathrm{b} \subseteq \precsim \mathrm{PCTL}_{\backslash \mathrm{X}}$ is by structural induction on the syntax of state formula $\varphi$ and path formula $\psi$ of safe $\mathrm{PCTL}_{\backslash \mathrm{x}}$, that is, we need to prove the following two results simultaneously.

(1) $r \models \varphi$ implies $s \models \varphi$ for any state formula $\varphi$ provided that $s \precsim^{\mathrm{b}} r$.

(2) $\omega_{2} \models \psi$ implies that $\omega_{1} \models \psi$ for any path formula $\psi$ provided that $\omega_{1} \precsim^{\mathrm{b}} \omega_{2}$.

We only consider $\varphi=\mathcal{P}_{\leq q}(\psi)$ where $\psi=\varphi_{1} \cup \varphi_{2}$ since the other cases are similar. Suppose that $r \models \varphi$, we need to prove that $s \models \varphi$. We proceed by contradiction, and assume that $s \not$ $\varphi$, then there exists $\sigma$ such that $\operatorname{Prob}_{\sigma, s}(\{\omega \mid \omega \models \psi\})>q$. By induction hypothesis $\operatorname{Sat}\left(\varphi_{1}\right)$ and $\operatorname{Sat}\left(\varphi_{2}\right)$ are $\precsim^{\mathrm{b}}$ downward closed, thus $\operatorname{Prob}_{\sigma, s}\left(\operatorname{Sat}\left(\varphi_{1}\right), \operatorname{Sat}\left(\varphi_{2}\right), s\right)=\operatorname{Prob}_{\sigma, s}(\{\omega \mid \omega \models$ $\psi\})>q$. Since $r \models \varphi$, there does not exist $\sigma^{\prime}$ such that $\operatorname{Prob} \sigma, r\left(\operatorname{Sat}\left(\varphi_{1}\right), \operatorname{Sat}\left(\varphi_{2}\right), r\right) \geq$ 
$\operatorname{Prob}_{\sigma, s}\left(\operatorname{Sat}\left(\varphi_{1}\right), \operatorname{Sat}\left(\varphi_{2}\right), s\right)$ which contradicts the assumption that $s \precsim^{\mathrm{b}} r$, thus $s \models \varphi$, and $s \precsim \mathrm{PCTL}_{\backslash \mathrm{x}} r$.

The weak simulation equivalent to $\precsim \mathrm{PCTL}_{\backslash_{\mathrm{X}}^{*}}^{*}$ can also be obtained in a straightforward way by adapting Definition 5.7 .

Definition 6.17. A relation $\mathcal{R} \subseteq S \times S$ is a weak simulation iff $s \mathcal{R} r$ implies that $L(s)=$ $L(r)$ and for any $\widetilde{\Omega} \subseteq\left(\mathcal{R}^{\downarrow}\right)^{+}$and any scheduler $\sigma$, there exists $\sigma^{\prime}$ such that $\operatorname{Prob}_{\sigma^{\prime}, r}\left(C_{\widetilde{\Omega}_{s t}}\right) \geq$ $\operatorname{Prob}_{\sigma, s}\left(C_{\widetilde{\Omega}_{s t}}\right)$.

We write $s \precsim r$ whenever there is a weak simulation $\mathcal{R}$ such that $s \mathcal{R} r$.

Again $\precsim$ is not compositional, but it coincides with $\precsim \mathrm{PCTL}_{\uparrow_{\mathrm{x}}}$, therefore we have the following theorem.

Theorem 6.18. $\precsim$ is a preorder, and $\precsim=\precsim \mathrm{PCTL}_{\mid \mathrm{X}}^{*}$.

Proof. The reflexivity of $\precsim$ is trivial. We prove the transitivity of $\precsim$. Suppose that $s \precsim r$ and $r \precsim t$, then for any $\widetilde{\Omega} \subseteq\left(\precsim^{\downarrow}\right)^{+}$and scheduler $\sigma$, there exists $\sigma^{\prime}$ such that $\operatorname{Prob}_{\sigma^{\prime}, r}\left(C_{\widetilde{\Omega}_{s t}}\right) \geq \operatorname{Prob}_{\sigma, s}\left(C_{\widetilde{\Omega}_{s t}}\right)$. Since we also have $r \precsim{ }^{b} t$, so there exists $\sigma^{\prime \prime}$ such that $\operatorname{Prob}_{\sigma^{\prime \prime}, t}\left(C_{\widetilde{\Omega}_{s t}}\right) \geq \operatorname{Prob}_{\sigma^{\prime}, r}\left(C_{\widetilde{\Omega}_{s t}}\right) \geq \operatorname{Prob}_{\sigma, s}\left(C_{\widetilde{\Omega}_{s t}}\right)$. This proves the transitivity of $\precsim$.

For the second statement let $\mathcal{R}=\left\{(s, r) \mid s \underset{\approx \mathrm{PCTL}_{\backslash \mathrm{x}}^{*}}{r}\right\}$ and $s \mathcal{R} r$, we need to prove that for any $\widetilde{\Omega} \subseteq\left(\mathcal{R}^{\downarrow}\right)^{+}$and scheduler $\sigma$, there exists a scheduler $\sigma^{\prime}$ such that $\operatorname{Prob}_{\sigma^{\prime}, r}\left(C_{\widetilde{\Omega}_{s t}}\right) \geq \operatorname{Prob}_{\sigma, s}\left(C_{\widetilde{\Omega}_{s t}}\right)$. By induction hypothesis $C_{\widetilde{\Omega}_{s t}}$ is $\mathcal{R}$ downward closed, thus there exists $\psi$ such that $\operatorname{Sat}(\psi)=C_{\widetilde{\Omega}_{s t}}$. We proceed by contradiction. Suppose that there does not exist $\sigma^{\prime}$ such that $\operatorname{Prob}_{\sigma^{\prime}, r}\left(C_{\widetilde{\Omega}_{s t}}\right) \geq \operatorname{Prob}_{\sigma, s}\left(C_{\widetilde{\Omega}_{s t}}\right)$, then there exists $q$ such that $r \models \mathcal{P}_{\leq q}(\psi)$, but $s \not \models \mathcal{P}_{\leq q}(\psi)$, which contradicts the assumption that $s \underset{\precsim \mathrm{PCTL}_{\backslash \mathrm{X}}^{*}}{r}$. Therefore there must exist a scheduler $\sigma^{\prime}$ such that $\operatorname{Prob}_{\sigma^{\prime}, r}\left(C_{\widetilde{\Omega}_{s t}}\right) \geq \operatorname{Prob}_{\sigma, s}\left(C_{\widetilde{\Omega}_{s t}}\right)$.

The proof of $\precsim \subseteq \precsim \mathrm{PCTL}_{\backslash \mathrm{X}}^{*}$ is by structural induction on the syntax of state formula $\varphi$ and path formula $\psi$ of safe $\mathrm{PCTL}_{\backslash \mathrm{x}}^{*}$, that is, we need to prove the following two results simultaneously.

(1) $r \models \varphi$ implies $s \models \varphi$ for any state formula $\varphi$ provided that $s \precsim r$.

(2) $\omega_{2} \models \psi$ implies that $\omega_{1} \models \psi$ for any path formula $\psi$ provided that $\omega_{1} \precsim \omega_{2}$.

We only consider $\varphi=\mathcal{P}_{\geq q}(\psi)$ since the other cases are similar. Suppose that $r \models \varphi$, we need to prove that $s \models \varphi$. We prove by contradiction, and assume that $s \not \models \varphi$, then there exists $\sigma$ such that $\operatorname{Prob}_{\sigma, s}(\{\omega \mid \omega \models \psi\})<q$. By induction hypothesis $\{\omega \mid \omega \models \psi\}$ is $\precsim$ downward closed, thus there exists $\widetilde{\Omega}_{s t}$ such that $C_{\widetilde{\Omega}_{s t}}=\{\omega \mid \omega \models \psi\}$. Since $r \models \varphi$, there does not exist $\sigma^{\prime}$ such that $\operatorname{Prob}_{\sigma, r}\left(C_{\widetilde{\Omega}_{s t}}\right) \geq \operatorname{Prob}_{\sigma, s}\left(C_{\widetilde{\Omega}_{s t}}\right)=q$ which contradicts the assumption that $s \precsim r$, thus $s \models \varphi$, and $s \underset{\precsim \mathrm{PCTL}_{\backslash \mathrm{x}}^{*} r}{ } r$.

6.4. Simulation kernels and summary. Let $\mathcal{R}^{-1}$ denote the reverse of $\mathcal{R}$, then $\mathcal{R} \cap \mathcal{R}^{-1}$ is the simulation kernel. In this section we will show the relation between the simulation kernels and their correspondent bisimulations. Not surprisingly, the simulation kernels are coarser than the bisimulations as shown in the following lemma:

Lemma 6.19.

(1) $\sim_{i}^{\mathrm{b}} \subset\left(\prec_{i}^{\mathrm{b}} \cap\left(\prec_{i}^{\mathrm{b}}\right)^{-1}\right)$. 
(2) $\sim_{i} \subset\left(\prec_{i} \cap \prec_{i}^{-1}\right)$.

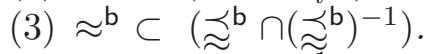

$(4) \approx \subset\left(\underset{\approx}{\gtrsim^{-1}}\right)$.

Proof. We only prove the first clause here, since the others are quite similar. The inclusion $\sim_{i}^{\mathrm{b}} \subseteq \prec_{i}^{\mathrm{b}} \cap\left(\prec_{i}^{\mathrm{b}}\right)^{-1}$ is straightforward. To show that the inclusion is strict, it is enough to give a counterexample. Suppose we have three states $s_{1}, s_{2}$, and $s_{3}$ such that $s_{1} \prec_{i}^{\mathrm{b}} s_{2} \prec_{i}^{\mathrm{b}} s_{3}$ but $s_{3} \quad \nprec_{i}^{\mathrm{b}} s_{2} \quad \nprec_{i}^{\mathrm{b}} s_{1}$. Let $s$ and $r$ be two states such that $L(s)=L(r)$. In addition $s$ has three transitions: $s \rightarrow \mathcal{D}_{s_{1}}, s \rightarrow \mathcal{D}_{s_{2}}, s \rightarrow \mathcal{D}_{s_{3}}$, and $r$ only has two transitions: $r \rightarrow \mathcal{D}_{s_{1}}$, $r \rightarrow \mathcal{D}_{s_{3}}$. Then it should be easy to check that $s \prec_{i}^{\mathrm{b}} r$ and $r \prec_{i}^{\mathrm{b}} s$, the only non-trivial case is when $s \rightarrow \mathcal{D}_{s_{2}}$. Since $s_{2} \prec_{i}^{\mathrm{b}} s_{3}$, thus there exists $r \rightarrow \mathcal{D}_{s_{3}}$ such that $\mathcal{D}_{s_{2}} \sqsubseteq \prec_{i}^{\mathrm{b}} \mathcal{D}_{s_{3}}$. But obviously $s \chi_{i}^{\mathrm{b}} r$, since the transition $s \rightarrow \mathcal{D}_{s_{2}}$ cannot be simulated by any transition of $r$.

The relationship of all the preorders is similar as for bisimulations, which we have summarized in Fig. 3 and 4 respectively. We could draw similar figures for the preorders by replacing all the equivalence relations by their correspondent preorders, but we omit them here for lack of space.

\section{Countable states}

Until now we have only considered PAs with finitely many states. In this section we will show that these results also apply for PAs with countable states. Assume $S$ is a countable set of states. We adopt the method used in [1] to deal with strong branching bisimulation. First we recall some standard notations from topology theory. Given a metric space $(S, d)$ where $d$ is a metric, a sequence $\left\{s_{i} \mid i \geq 0\right\}$ converges to $s$ iff for any $\varepsilon>0$, there exists $n$ such that $d\left(s_{m}, s\right)<\varepsilon$ for any $m \geq n$. A metric space $(S, d)$ is compact if every infinite sequence has a convergent subsequence to an element in $S$.

Below follows the definition of metric over distributions: Refer to [1] for more details.

Definition 7.1. Given two distributions $\mu, \nu \in \operatorname{Dist}(S)$, the metric $d$ is defined by $d(\mu, \nu)=$ $\operatorname{Sup}_{C \subseteq S}|\mu(C)-\nu(C)|$.

Since the metric is defined over distributions, we need to adapt the definition of $\operatorname{Prob}_{\sigma, s}\left(C, C^{\prime}, n, s\right)$ in the following way: $s \stackrel{n, C}{\Longrightarrow} \mu$ iff either i) $\mu=\mathcal{D}_{s}$, or ii) $s \rightarrow \nu$ such that

$$
\sum_{\forall r \in \operatorname{Supp}(\nu) . r \stackrel{n-1, C}{\Longrightarrow} \nu_{r}} \nu(r) \cdot \nu_{r}=\mu .
$$

Obviously for each $\sigma, C, C^{\prime}$, and $n$, there exists $s \stackrel{n, C}{\Longrightarrow} \mu$ such that $\mu\left(C^{\prime}\right)=\operatorname{Prob}_{\sigma, s}\left(C, C^{\prime}, n, s\right)$.

Now we can define the compactness of PAs as in [11] with a slight difference.

Definition 7.2. Given a PA $\mathcal{P}, \mathcal{P}$ is $i$-compact iff the metric space $(\{\mu \mid s \stackrel{i, C}{\Longrightarrow} \mu\}, d)$ is compact for each $s \in S$ and $\sim_{i}^{\mathrm{b}}$ closed set $C$.

As mentioned in [11, 33], the convex closure does not change the compactness, thus we can extend $\stackrel{n, C}{\Longrightarrow}$ to allow combined transitions in a standard way without changing anything, but for simplicity we omit this. A PA is compact iff it is $i$-compact for any $i \geq 1$.

We introduce the definition of capacity as follows. 
Definition 7.3. Given a set of states $S$ and a $\sigma$-algebra $\mathcal{B}$, a capacity on $\mathcal{B}$ is a function Cap $: \mathcal{B} \rightarrow R^{\geq 0}$ such that:

(1) $\operatorname{Cap}(\emptyset)=0$,

(2) whenever $C_{1} \subseteq C_{2}$ with $C_{1}, C_{2} \in \mathcal{B}$, then $\operatorname{Cap}\left(C_{1}\right) \leq \operatorname{Cap}\left(C_{2}\right)$,

(3) whenever there exists $C_{1} \subseteq C_{2} \subseteq \ldots$ such that $\cup_{i \geq 1} C_{i}=C$, or $C_{1} \supseteq C_{2} \supseteq \ldots$ such that $\cap_{i \geq 1} C_{i}=C$, then $\lim _{i \rightarrow \infty} \operatorname{Cap}\left(C_{i}\right)=\operatorname{Cap}(C)$.

A capacity $C a p$ is sub-additive iff $\operatorname{Cap}\left(C_{1} \cup C_{2}\right) \leq \operatorname{Cap}\left(C_{1}\right)+\operatorname{Cap}\left(C_{2}\right)$ for any $C_{1}, C_{2} \in \mathcal{B}$.

Different from [11], the value of $\operatorname{Prob}_{\sigma, s}\left(C, C^{\prime}, n, s\right)$ depends on both $C$ and $C^{\prime}$. Let $\operatorname{PreCap}_{s, n}^{C}\left(C^{\prime}\right)=\operatorname{Sup}_{\sigma} \operatorname{Prob}_{\sigma, s}\left(C, C^{\prime}, n, s\right)$ and $\operatorname{PostCap}_{s, n}^{C^{\prime}}(C)=\operatorname{Sup}_{\sigma} \operatorname{Prob}_{\sigma, s}\left(C, C^{\prime}, n, s\right)$ i.e. given a $C^{\prime}$, PreCap $C_{s, n}^{C}$ will return the maximum probability from $s$ to $C^{\prime}$ in at most $n$ steps via only states in $C$, similar for $\operatorname{PostCap}_{s, n}^{C^{\prime}}$. The following lemma shows that both PreCap $C_{s, n}^{C}$ and PostCap $C_{s, n}^{C^{\prime}}$ are sub-additive capacities.

Lemma 7.4. PreCap ${ }_{s, n}^{C}$ and PostCap ${ }_{s, n}^{C^{\prime}}$ are sub-additive capacities on $\mathcal{B}$ where $\mathcal{B}$ is the $\sigma$-algebra only containing $\sim_{n}^{\mathrm{b}}$ closed sets.

Proof. Refer to the proof of Lemma 5.2 in [11].

Now we can show that the following results are still valid as long as the given PA is compact even if it contains infinitely countable states.

Theorem 7.5. Given a compact PA,

(1) $\sim_{n}^{\mathrm{b}}=\sim_{\mathrm{PCTL}_{n}^{-}}$

(2) there exists $n \geq 0$ such that $\sim_{n}^{\mathrm{b}}=\sim_{\mathrm{PCTL}}$.

Proof.

(1) $\sim_{n}^{\mathrm{b}}=\sim_{\mathrm{PCTL}_{n}^{-}}$:

The proof of $\sim_{n}^{\mathrm{b}} \subseteq \sim_{\mathrm{PCTL}_{n}^{-}}$is similar with the proof of Theorem 4.7, and is omitted here. We prove that $\sim_{\mathrm{PCTL}_{n}^{-}} \subseteq \sim_{n}^{\mathrm{b}}$ in the sequel following the proof of Theorem 6.10 in [11. Let $\mathcal{R}=\left\{(s, r) \mid s \sim_{\mathrm{PCTL}_{n}^{-}} r\right\}$, we need to prove that $\mathcal{R}$ is a strong $n$-depth branching bisimulation. In order to do so, we need to prove that for any $(s, r) \in \mathcal{R}$, if $\operatorname{Prob}_{\sigma, s}\left(C, C^{\prime}, n, s\right)>0$ for some $\sigma$, there exists $\sigma^{\prime}$ such that $\operatorname{Prob}_{\sigma^{\prime}, r}\left(C, C^{\prime}, n, r\right) \geq \operatorname{Prob}_{\sigma, s}\left(C, C^{\prime}, n, s\right)$ and vice versa. This is equivalent to say that $\operatorname{Sup}_{\sigma} \operatorname{Prob}_{\sigma, s}\left(C, C^{\prime}, n, s\right)=\operatorname{Sup}_{\sigma} \operatorname{Prob}_{\sigma, r}\left(C, C^{\prime}, n, s\right)$, i.e. $\operatorname{PreCap}{ }_{s, n}^{C}\left(C^{\prime}\right)=\operatorname{PreCap}_{r, n}^{C}\left(C^{\prime}\right)$ (or equivalently PostCap $\mathrm{C}_{s, n}^{C^{\prime}}(C)=\operatorname{PostCap}_{r, n}^{C^{\prime}}(C)$ ) for each $\mathcal{R}$ closed sets $C$ and $C^{\prime}$. Since both $C$ and $C^{\prime}$ may be countable union of equivalence classes where each equivalence class can only be characterized by countable many formulas, therefore we have $C=\cup_{i=1}^{\infty}\left(\cap_{j=1}^{\infty} C_{i, j}\right)$ and $C^{\prime}=\cup_{i=1}^{\infty}\left(\cap_{j=1}^{\infty} C_{i, j}^{\prime}\right)$ where $\cap_{j=1}^{\infty} C_{i, j}$ corresponds to the $i$-th equivalence class in $C$, and $C_{i, j}$ corresponds to the set of states determining by the $j$-th formula satisfied by $i$-th equivalence class, similar for $\cap_{j=1}^{\infty} C_{i, j}^{\prime}$ and $C_{i, j}^{\prime}$. Let $B_{k}=$ $\cap_{j=1}^{\infty}\left(\cup_{i=1}^{k} C_{i, j}\right), A_{k}^{l}=\cap_{j=1}^{l}\left(\cup_{i=1}^{k} C_{i, j}\right)$, and $B_{k}^{\prime}=\cap_{j=1}^{\infty}\left(\cup_{i=1}^{k} C_{i, j}^{\prime}\right), A_{k}^{l}=\cap_{j=1}^{l}\left(\cup_{i=1}^{k} C_{i, j}^{\prime}\right)$. It is easy to see that $B_{k}$ and $B_{k}^{\prime}$ are increasing sequences of $\mathcal{R}$ closed sets such that $\cup_{k=1}^{\infty} B_{k}=C$, and $\cup_{k=1}^{\infty} B_{k}^{\prime}=C^{\prime}$, while $A_{k}^{l}$ and $A_{k}^{\prime l}$ are decreasing sequences of $\mathcal{R}$ closed sets such that $\cap_{l=1}^{\infty} A_{k}^{l}=B_{k}$ and $\cap_{l=1}^{\infty} A_{k}^{\prime l}=B_{k}^{\prime}$. Both $A_{k}^{l}$ and $A_{k}^{\prime l}$ only contain conjunction and disjunction of finite formulas, thus can be described by $\mathrm{PCTL}_{n}^{-}$, that is, let $\varphi=\wedge_{j=1}^{l}\left(\vee_{i=1}^{k} \varphi_{C_{i, j}}\right)$ and $\varphi^{\prime}=\wedge_{j=1}^{l}\left(\vee_{i=1}^{k} \varphi_{C_{i, j}^{\prime}}\right)$ where $\varphi_{C_{i, j}}$ denotes the $j$-th formula 
satisfied by the $i$-th equivalence class in $C$, similarly for $\varphi_{C_{i, j}^{\prime}}$. Obviously, we have $\operatorname{Sat}(\varphi)=A_{k}^{l}$ and $\operatorname{Sat}\left(\varphi^{\prime}\right)=A_{k}^{\prime l}$.

Assume that $q=\operatorname{PreCap} A_{r, n}^{A_{k}^{l}}\left(A_{k}^{\prime l}\right)<\operatorname{PreCap} A_{s, n}^{A_{k}^{l}}\left(A_{k}^{\prime l}\right)=p$, then it holds that $r \models$ $\mathcal{P}_{\leq q}\left(\varphi \bigcup^{\leq n} \varphi^{\prime}\right)$, but $s \not \forall \mathcal{P}_{\leq q}\left(\varphi \bigcup^{\leq n} \varphi^{\prime}\right)$, which contradicts the fact that $s \sim_{\mathrm{PCTL}_{n}^{-}} r$. Therefore $\operatorname{PreCap}_{s, n}^{A_{k}^{l}}\left(A_{k}^{\prime l}\right)=\operatorname{PreCap}_{r, n}^{A_{k}^{l}}\left(A_{k}^{\prime l}\right)$ for each $l$ and $k$. By Definition 7.3 and Lemma 7.4, we know that $\operatorname{PreCap}_{s, n}^{A_{k}^{l}}\left(C^{\prime}\right)=\operatorname{Pre} \operatorname{Cap}_{r, n}^{A_{k}^{l}}\left(C^{\prime}\right)$ for each $l$ and $k$. Note that $\operatorname{PreCap} A_{s, n}^{A_{k}^{l}}\left(C^{\prime}\right)=\operatorname{PostCap}_{s, n}^{C^{\prime}}\left(A_{k}^{l}\right)$, thus PostCap $C_{s, n}^{C^{\prime}}\left(A_{k}^{l}\right)=\operatorname{PostCap}_{r, n}^{C^{\prime}}\left(A_{k}^{l}\right)$ for each $l$ and $k$, again by Definition 7.3 and Lemma 17.4, we conclude that PostCap ${ }_{s, n}{ }^{\prime}(C)=$ PostCap ${ }_{r, n}^{C^{\prime}}(C)$.

(2) $\exists n \geq 0 . \stackrel{\sim}{\mathrm{b}}=\sim_{\mathrm{PCTL}}$ :

Suppose that $\sim$ PCTL $\subset \sim_{n}^{\mathrm{b}}$ for any $n \geq 0$ which means that there exist $s$ and $r$ such that $s \sim_{n}^{\mathrm{b}} r$ for any $n \geq 0$, but $s \nsim_{\mathrm{PCTL}} r$. As a result there exists $C, C^{\prime}$ and $\sigma$ such that $\lim _{i \rightarrow \infty} \operatorname{Prob}_{\sigma, s}\left(C, C^{\prime}, i, s\right)>0$, but there does not exist $\sigma^{\prime}$ such that $\lim _{i \rightarrow \infty} \operatorname{Prob}_{\sigma^{\prime}, r}\left(C, C^{\prime}, i, r\right) \geq \lim _{i \rightarrow \infty} \operatorname{Prob}_{\sigma, s}\left(C, C^{\prime}, i, s\right)$. In other words, $\lim _{i \rightarrow \infty} \operatorname{Prob}_{\sigma^{\prime}, r}\left(C, C^{\prime}, i, r\right)<\lim _{i \rightarrow \infty} \operatorname{Prob}_{\sigma, s}\left(C, C^{\prime}, i, s\right)$ for any $\sigma^{\prime}$ which indicates that there exists $n \geq 0$ such that $\operatorname{Prob}_{\sigma^{\prime}, r}\left(C, C^{\prime}, n, r\right)<\operatorname{Prob}_{\sigma, s}\left(C, C^{\prime}, n, s\right)$ for any $\sigma^{\prime}$, therefore $s \nsim_{\mathrm{PCTL}_{i}^{-}} r$ which contradicts our assumption.

In a similar way we can extend the results of this section to strong bisimulations and weak bisimulations: We skip the proofs here. For the simulations, we need to do more work, since there may be uncountably many downward closed sets. For a relation $\mathcal{R}$ over $S$, let $\equiv_{\mathcal{R}}$ denote the largest equivalence relation contained in the reflexive and transitive closure of $(\mathcal{R} \cup I D)$. The following lemma states that a downward closed set can be expressed as a union of equivalence classes:

Lemma 7.6. Let $\mathcal{R} \subseteq S \times S$ be a relation, and $C \subseteq S$ be a $\mathcal{R}$ downward closed set, then $C$ is a union of equivalence classes of $\equiv_{\mathcal{R}}$.

The above lemma is a slight generalization of Lemma 5.1 in [20] with only two differences: i) we consider downward closed sets instead of upward closed sets, ii) we do not require $\mathcal{R}$ to be a preorder, but these do not change the proof there.

Given a $\mathcal{R}$ downward closed set $C$, we say $C$ is finitely generated if there exists a finite set of equivalence classes of $\left\{C_{i} \in S / \equiv_{\mathcal{R}}\right\}_{i \in I}$ such that $C=\cup_{i \in I} C_{i}$. Since the set of the equivalence classes in $S / \equiv_{\mathcal{R}}$ is countable, thus the set of finitely generated $\mathcal{R}$ downward closed set is also countable [20]. The following lemma shows an alternative definition of $\prec_{i}^{\mathrm{b}}$ in Definition 6.4 where we only focus on finitely generated downward closed sets:

Lemma 7.7. A relation $\mathcal{R} \subseteq S \times S$ is a strong $i$-depth branching simulation with $i \geq 1$ iff $s \mathcal{R} r$ implies that $s \prec_{i-1}^{\mathrm{b}} r$ and for any finitely generated $\mathcal{R}$ downward closed sets $C, C^{\prime}$, and any scheduler $\sigma$, there exists $\sigma^{\prime}$ such that $\operatorname{Prob}_{\sigma^{\prime}, r}\left(C, C^{\prime}, i, r\right) \geq \operatorname{Prob}_{\sigma, s}\left(C, C^{\prime}, i, s\right)$.

We write $s \prec_{i}^{\mathrm{b}} r$ whenever there is a strong $i$-depth branching simulation $\mathcal{R}$ such that $s \mathcal{R} r$.

Proof. The proof is similar as the proof of Lemma 5.2 in [20]. Let $\left(\prec_{i}^{\mathrm{b}}\right)^{\prime}$ denote the new definition, we need to prove that $s \quad \prec_{i}^{\mathrm{b}} \quad r$ iff $s\left(\prec_{i}^{\mathrm{b}}\right)^{\prime} r$. Since finitely generated $\mathcal{R}$ downward closed sets are special cases of $\mathcal{R}$ downward closed sets, therefore $s \quad \prec_{i}^{\mathrm{b}} r$ 
implies $s\left(\prec_{i}^{\mathrm{b}}\right)^{\prime} r$. We prove that $s\left(\prec_{i}^{\mathrm{b}}\right)^{\prime} r$ implies $s \quad \prec_{i}^{\mathrm{b}} r$ by contradiction. Suppose that for any finitely generated $\mathcal{R}$ downward closed sets $C, C^{\prime}$ and $\sigma$, there exists $\sigma^{\prime}$ such that $\operatorname{Prob}_{\sigma^{\prime}, r}\left(C, C^{\prime}, i, r\right) \geq \operatorname{Prob}_{\sigma, s}\left(C, C^{\prime}, i, s\right)$, but there exists $\mathcal{R}$ downward closed sets $C, C^{\prime}$ and $\sigma$ such that $\operatorname{Prob}_{\sigma^{\prime}, r}\left(C, C^{\prime}, i, r\right)<\operatorname{Prob}_{\sigma, s}\left(C, C^{\prime}, i, s\right)$ for any $\sigma^{\prime}$. Let $\sigma$ be a scheduler such that $\operatorname{Prob}_{\sigma^{\prime}, r}\left(C, C^{\prime}, i, r\right)<\operatorname{Prob}_{\sigma, s}\left(C, C^{\prime}, i, s\right)$ for any $\sigma^{\prime}$ and $\varepsilon=$ $\operatorname{Prob}_{\sigma, s}\left(C, C^{\prime}, i, s\right)-\operatorname{Prob}_{\sigma^{\prime}, r}\left(C, C^{\prime}, i, r\right)>0$. According to Lemma 7.6, there exists sets of equivalences classes: $\left\{C_{j} \in S / \equiv_{\mathcal{R}}\right\}_{j \in J}$ and $\left\{C_{k} \in S / \equiv_{\mathcal{R}}\right\}_{k \in K}$ such that $C=\cup_{j \in J} C_{i}$ and $C^{\prime}=\cup_{k \in K} C_{k}$ where $J, K$ are (infinite) sets of indexes. Define two sequences of finitely generated $\mathcal{R}$ downward closed sets: $\left\{C_{\leq j}=\cup_{j^{\prime} \in J \wedge j^{\prime} \leq j} C_{j^{\prime}}\right\}_{j \in J},\left\{C_{\leq k}=\cup_{k^{\prime} \in K \wedge k^{\prime} \leq k} C_{k^{\prime}}\right\}_{k \in K}$. Obviously both $\operatorname{Prob}_{\sigma, s}\left(C, C_{\leq k}, i, s\right)$ and $\operatorname{Prob}_{\sigma, s}\left(C_{\leq j}, C^{\prime}, i, s\right)$ are monotone, non-decreasing and converge to $\operatorname{Prob}_{\sigma, s}\left(C, C^{\prime}, i, s\right)$ for any $C$ and $C^{\prime}$. Therefore there exists $j \in J$ and $k \in K$ such that

This implies

$$
\begin{gathered}
\operatorname{Prob}_{\sigma, s}\left(C_{\leq j}, C^{\prime}, i, s\right)>\operatorname{Prob}_{\sigma, s}\left(C, C^{\prime}, i, s\right)-\frac{\varepsilon}{4}, \text { and } \\
\operatorname{Prob}_{\sigma, s}\left(C_{\leq j}, C_{\leq k}, i, s\right)>\operatorname{Prob}_{\sigma, s}\left(C_{\leq j}, C^{\prime}, i, s\right)-\frac{\varepsilon}{4} .
\end{gathered}
$$

$$
\begin{gathered}
\operatorname{Prob}_{\sigma, s}\left(C_{\leq j}, C_{\leq k}, i, s\right)>\operatorname{Prob}_{\sigma, s}\left(C, C^{\prime}, i, s\right)-\frac{\varepsilon}{2} \\
=\operatorname{Prob}_{\sigma^{\prime}, r}\left(C, C^{\prime}, i, r\right)+\frac{\varepsilon}{2}>\operatorname{Prob}_{\sigma^{\prime}, r}\left(C, C^{\prime}, i, r\right) \geq \operatorname{Prob}_{\sigma, s}\left(C_{\leq j}, C_{\leq k}, i, s\right),
\end{gathered}
$$

which contradicts the assumption.

By Lemma 7.7 it is enough to consider all the finitely generated $\prec_{i}^{\mathrm{b}}$ downward closed sets in Definition 7.2 which is countable. The extension of Theorem 6.6 to the countable state space is then routine i.e. we should define the capacity as in Definition 7.3, and then show that finite formulas are enough to characterize $\operatorname{Prob}_{\sigma^{\prime}, s}\left(C, C^{\prime}, i, s\right)$ even if $C$ and $C^{\prime}$ are countable infinite. Moreover the definitions of other variants of simulations in Section 6 can be adopted to only consider finitely generated downward closed sets too, thus their logic characterizations can also be extended to countable states.

\section{The COARSEST CONGRUENT Bisimulations AND Simulations}

Before we have shown that $\sim_{P}$ is a congruence but cannot be characterized by $\sim_{P C T L}$ since it is too fine. On the other hand, $\sim^{\mathrm{b}}$ can be characterized by $\sim_{\mathrm{PCTL}}$, but it is not a congruence in general. This indicates that $\sim_{P C T L}$ is not a congruence. Therefore a natural question one may ask is what is the largest subset of $\sim_{\mathrm{PCTL}}$ which is congruent. The following theorem shows that $\sim_{P}$ is the coarsest congruence relation in $\sim_{P C T L}$ provided that the given PA is compact.

Theorem 8.1. Given a compact $\mathrm{PA}, \sim_{\mathrm{P}}$ is the coarsest congruence relation in $\sim_{\mathrm{PCTL}}$.

Proof. We proceed by contradiction. Suppose that there exists a congruence $\simeq \subset \sim_{\mathrm{PCTL}}$. Suppose that there exists $s$ and $r$ such that $s \simeq r$ but $s \nsim_{\mathrm{P}} r$. According to Definition 3.3 there exists $s \rightarrow \mu$ such that there does not exist $r \rightarrow_{\mathrm{P}} \nu$ with $\mu \sim_{\mathrm{P}} \nu$. The idea is to show that there always exists $t$ such that $s\left\|t \chi_{\mathrm{PCTL}} r\right\| t$ in this case, then it is enough to give a formula $\varphi$ such that $r \| t \models \varphi$, but $s \| t \not \models \varphi$.

Let $\operatorname{Supp}(\mu)=\left\{s_{1}, s_{2}, \ldots\right\}$ and $\mu\left(s_{i}\right)=a_{i}$ with $i \geq 1$, where we assume that $s_{i}(i \geq 1)$ belong to different equivalence classes for simplicity. Without loss of generality we assume that there exists $s \rightarrow \mu$ such that for any two (combined) transitions of $r: r \rightarrow_{\mathrm{p}} \nu_{1}$ and 
$r \rightarrow \mathrm{P} \nu_{2}$, there does not exist $0 \leq w_{1}, w_{2} \leq 1$ such that $w_{1}+w_{2}=1$ and $\mu \sim_{\mathrm{P}}\left(w_{1} \cdot \nu_{1}+w_{2} \cdot \nu_{2}\right)$ (every combined transition of $r$ can be seen as a combined transition of two other combined transitions of $r$ ). Let $\nu_{1}\left(s_{i}\right)=b_{i}$ and $\nu_{2}\left(s_{i}\right)=c_{i}$ in the following, then there must exist $i \neq j \geq 1$ such that there is no $0 \leq w_{1}, w_{2} \leq 1$ with $w_{1} \cdot b_{i}+w_{2} \cdot c_{i}=a_{i}, w_{1} \cdot b_{j}+w_{2} \cdot c_{j}=a_{j}$, and $w_{1}+w_{2}=1$, otherwise we will have $\mu \sim_{\mathrm{p}}\left(w_{1} \cdot \nu_{1}+w_{2} \cdot \nu_{2}\right)$ which contradicts the assumption.

Most of the cases are simple, for instance if $a_{i}>b_{i}, c_{i}, r$ will evolve into $s_{i}$ with probability less than $a_{i}$ which is not the case for $s$, thus $s \nsim_{\mathrm{PCTL}} r$ which contradicts the assumption. We only consider in detail the case when $c_{i}>b_{i}, b_{j}>c_{j}, a_{i} \in\left(b_{i}, c_{i}\right)$ and $a_{j} \in\left(c_{j}, b_{j}\right)$. Suppose that $\frac{b_{j}-a_{j}}{a_{i}-b_{i}}=\frac{a_{j}-c_{j}}{c_{i}-a_{i}}$, which implies that $\frac{a_{i}-b_{i}}{c_{i}-a_{i}}=\frac{b_{j}-a_{j}}{a_{j}-c_{j}}$. Let $w_{1}=\frac{1}{k+1}$ and $w_{2}=\frac{k}{k+1}$ where $k=\frac{a_{i}-b_{i}}{c_{i}-a_{i}}$, then it holds that $w_{1} \cdot b_{i}+w_{2} \cdot c_{i}=a_{i}$ and $w_{1} \cdot b_{j}+w_{2} \cdot c_{j}=a_{j}$, which contradicts the assumption. Therefore we have either $\frac{b_{j}-a_{j}}{a_{i}-b_{i}}<\frac{a_{j}-c_{j}}{c_{i}-a_{i}}$ or $\frac{b_{j}-a_{j}}{a_{i}-b_{i}}>\frac{a_{j}-c_{j}}{c_{i}-a_{i}}$. We only consider the case when $\frac{b_{j}-a_{j}}{a_{i}-b_{i}}>\frac{a_{j}-c_{j}}{c_{i}-a_{i}}$, since the other case is similar. Let $\rho_{1}$ and $\rho_{2}$ be two variables with values in $[0,1]$ such that

then we can see that:

$$
\frac{b_{j}-a_{j}}{a_{i}-b_{i}} \cdot \rho_{2}>\rho_{1}>\frac{a_{j}-c_{j}}{c_{i}-a_{i}} \cdot \rho_{2},
$$

$$
\begin{aligned}
& a_{i} \cdot \rho_{1}+a_{j} \cdot \rho_{2}<b_{i} \cdot \rho_{1}+b_{j} \cdot \rho_{2}, \\
& a_{i} \cdot \rho_{1}+a_{j} \cdot \rho_{2}<c_{i} \cdot \rho_{1}+c_{j} \cdot \rho_{2} .
\end{aligned}
$$

In other words, there exists $\rho_{1}$ and $\rho_{2}$ such that $a_{i} \cdot \rho_{1}+a_{j} \cdot \rho_{2}$ is smaller than $b_{i} \cdot \rho_{1}+b_{j} \cdot \rho_{2}$ and $c_{i} \cdot \rho_{1}+c_{j} \cdot \rho_{2}$.

Let $t$ be a state such that it can only evolve into $t_{1}$ with probability $\rho_{1}$ and $t_{2}$ with probability $\rho_{2}$ where $\rho_{1}+\rho_{2}=1$ and $\rho_{1} \in\left(\frac{a_{j}-c_{j}}{c_{i}-a_{i}} \cdot \rho_{2}, \frac{b_{j}-a_{j}}{a_{i}-b_{i}} \cdot \rho_{2}\right)$; obviously such $t$ always exists. Assume that all the states have distinct labels except for $s$ and $r$, moreover let

$$
\psi=\left(\left(L(s \| t) \vee L\left(s_{i} \| t\right) \vee\left(L\left(s_{j} \| t\right)\right)\right) \mathrm{U}^{\leq 2}\left(L\left(s_{i} \| t_{1}\right) \vee L\left(s_{j} \| t_{2}\right)\right)\right),
$$

it is not hard to see that the minimum probability of the paths of $s \| t$ satisfying $\psi$ is $a_{i} \cdot \rho_{1}+a_{j} \cdot \rho_{2}$ i.e. when $s \| t$ first performs the transition $s \rightarrow \mu$ of $s$ and then performs the transition $t \rightarrow\left\{\rho_{1}: t_{1}, \rho_{2}: t_{2}\right\}$ of $t$. Let $r \rightarrow_{\mathrm{p}} \nu=w_{1} \cdot \nu_{1}+w_{2} \cdot \nu_{2}$ be a transition for some $w_{1}$ and $w_{2}$ such that after performing it, the probability of the set of paths of $r \| t$ satisfying $\psi$ is minimal. It holds:

$$
\begin{aligned}
\nu\left(s_{i}\right) \cdot \rho_{1}+\nu\left(s_{j}\right) \cdot \rho_{2} & =\left(w_{1} \cdot b_{i}+w_{2} \cdot c_{i}\right) \cdot \rho_{1}+\left(w_{1} \cdot b_{j}+w_{2} \cdot c_{j}\right) \cdot \rho_{2} \\
& =w_{1} \cdot\left(b_{i} \cdot \rho_{1}+b_{j} \cdot \rho_{2}\right)+w_{2} \cdot\left(c_{i} \cdot \rho_{1}+c_{j} \cdot \rho_{2}\right) \\
& >a_{i} \cdot \rho_{1}+a_{j} \cdot \rho_{2},
\end{aligned}
$$

therefore we have $r \| t \models \mathcal{P}_{\geq q}(\psi)$ but $s \| t \not \models \mathcal{P}_{\geq q}(\psi)$ where $q=\nu\left(s_{i}\right) \cdot \rho_{1}+\nu\left(s_{j}\right) \cdot \rho_{2}$. In other words $s\left\|t \nsim_{\text {PCTL }} r\right\| t$, as a result $s\|t \not z r\| t$, so $\simeq$ is not a congruence.

When all the states do not have distinct labels, we can always construct formulas to distinguish them, since the PA is compact and these states are in different equivalence classes by assumption. The subsequent proof is then similar. This completes our proof. 
Theorem 8.1 can be extended to identify the coarsest congruent weak bisimulation in $\sim_{\mathrm{PCTL}} \mathrm{X}_{\mathrm{X}}$, and the coarsest congruent strong and weak simulations in $\prec \mathrm{PCTL}$ and $\precsim \mathrm{PCTL} \backslash \mathrm{X}$ respectively.

\section{Theorem 8.2.}

(1) $\simeq_{\mathrm{P}}$ is the coarsest congruence relation in $\sim_{\mathrm{PCTL}} \mathrm{X}_{\mathrm{X}}$,

(2) $\prec_{\mathrm{P}}$ is the coarsest congruent preorder in $\prec \mathrm{PCTL}$,

(3) $\precsim \mathrm{P}$ is the coarsest congruent preorder in $\precsim \mathrm{PCTL} \backslash \mathrm{x}$.

Proof. The proof is similar to the proof of Theorem 8.1 and we only sketch the proof of Clause (2) here. According to Lemma 5.2 in [20, $\mu \mathcal{R} \nu$ iff for each finitely generated $\mathcal{R}$ downward closed set $C, \mu(C) \leq \nu(C)$ where $\mathcal{R}$ is a preorder. In order to prove that $\prec_{\mathrm{p}}$ is the coarsest congruent preorder in $\prec \mathrm{PCTL}$, we need to show that for any relation $\preceq$ such that $\prec_{\mathrm{P}} \subset \preceq \subset \prec \mathrm{PCTL}$, it holds that $\preceq$ is not congruent, i.e. there exist $s, r$, and $t$ such that $s \preceq r$, but $s\|t \npreceq r\| t$. First assume that $\preceq$ is a congruence, and we then prove by contradiction as in Theorem 8.1 and show that if $s \preceq r$ and $s k_{\mathrm{P}} r$, there exists $t$ such that $s\left\|t \nprec_{\mathrm{PCTL}} r\right\| t$, thus $s\|t \npreceq r\| t$ which contradicts the assumption that $\preceq$ is a congruence. Since $s \nprec_{\mathrm{P}} r$, then there exists $s \rightarrow \mu$ such that there does not exist $r \rightarrow_{\mathrm{P}} \nu$ with $\mu \sqsubseteq \prec_{\mathrm{p}} \nu$. With the same argument as in Theorem 8.1 and Lemma 5.2 in [20], there exist $t$ and $\psi$ such that $r \| t \models \mathcal{P}_{\geq q}(\psi)$ but $s \| t \not \models \mathcal{P}_{\geq q}(\psi)$ i.e. $s \| t \nprec$ PCTL $r \| t$, thus $\preceq$ is not congruent.

\section{RELATED WORK}

For Markov chains, i.e., deterministic PAs, the logic PCTL characterizes bisimulations, and PCTL without $X$ operator characterizes weak bisimulations [15, 5]. As pointed out in [35], probabilistic bisimulation is sound, but not complete for PCTL over PAs. In the literature, various extensions of the Hennessy-Milner logic [18] are considered for characterizing bisimulations. Larsen and Skou [25] considered such an extension of Hennessy-Milner logic, which characterizes bisimulation for reactive probabilistic processes [25]. Similar results are further studied for labelled Markov processes [28, 11] (with continuous state space). For PAs, Jonsson et al. [23] considered a two-sorted logic in the Hennessy-Milner style to characterize strong bisimulations. In [20], the results are also extended to characterize simulations.

Weak bisimulation was first defined in the context of PAs by Segala and Lynch [35, and then formulated for alternating models by Philippou et al. [30. The seemingly very related work is by Desharnais et al. [11], where it is shown that PCTL* is sound and complete with respect to weak bisimulation for alternating automata. The key difference is that the model they have considered is not the same as PAs considered in this paper. Briefly, in alternating automata, states are either non-deterministic like in transition systems, or stochastic like in discrete-time Markov chains. As discussed in [36], a PA can be transformed to an alternating automaton by replacing each transition $s \rightarrow \mu$ by two consecutive transitions $s \rightarrow s^{\prime}$ and $s^{\prime} \rightarrow \mu$ where $s^{\prime}$ is the new inserted state. Surprisingly, for alternating automata, Desharnais et al. have shown that weak bisimulation - defined in the standard manner - characterizes PCTL* formulas. The following example illustrates why it works in that setting, but fails for PAs.

Example 9.1. Refer to Fig. 1, we need to add three additional states $s_{\mu_{1}}, s_{\mu_{2}}$, and $s_{\mu_{3}}$ in order to transform $s$ and $r$ to states in an alternating automaton. The resulting automaton 

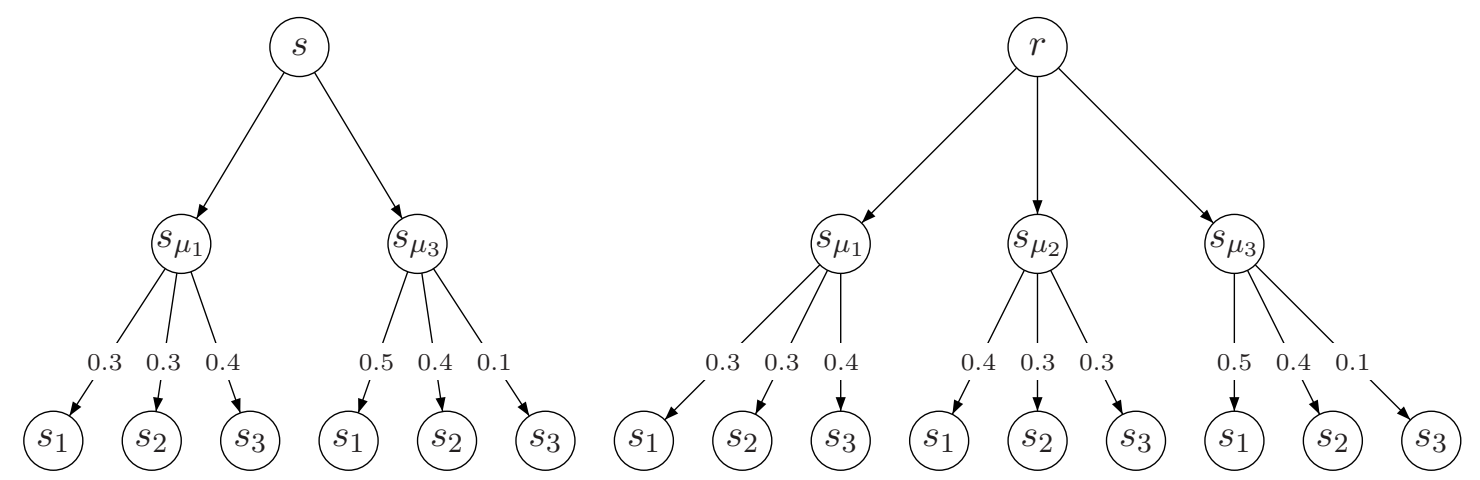

Figure 6: Alternating automata.

is shown in Fig. 6. Suppose that $s_{1}, s_{2}$, and $s_{3}$ are three absorbing states with different atomic propositions, so they are not (weak) bisimilar, as a result $s_{\mu_{1}}, s_{\mu_{2}}$ and $s_{\mu_{3}}$ are not (weak) bisimilar either since they can evolve into $s_{1}, s_{2}$, and $s_{3}$ with different probabilities. Therefore $s$ and $r$ are not (weak) bisimilar. Let $\varphi=\mathcal{P}_{\geq 0.4}\left(\mathbf{X} L\left(s_{1}\right)\right) \wedge \mathcal{P}_{\geq 0.3}\left(\mathbf{X} L\left(s_{2}\right)\right) \wedge$ $\mathcal{P}_{\geq 0.3}\left(\mathrm{X} L\left(s_{3}\right)\right)$, it is not hard to see that $s_{\mu_{2}} \models \varphi$ but $s_{\mu_{1}}, s_{\mu_{3}} \vDash \varphi$, so $s \models \overline{\mathcal{P}}_{\leq 0}(\mathbf{X} \varphi)$ while $r \not \forall \mathcal{P}_{\leq 0}(\mathbf{X} \varphi)$. When working in the setting of PAs, $s_{\mu_{1}}, s_{\mu_{2}}$, and $s_{\mu_{3}}$ will not be considered as states, so we cannot use the above arguments for alternating automata any more.

In the definition of $\sim_{1}$ and $\prec_{1}$, we choose first the downward closed set $C$ before the successor distributions to be matched, which is the key for achieving our new notions of bisimulations and simulations. This approach was first adopted in [9] to define the a priori metric for Markov decision processes, where it was shown that the a priori metric can be characterized by the quantitative $\mu$-calculus. In [13] this approach was also used to define a priori $\varepsilon$-bisimulation and simulation relations.

\section{Conclusion And Future WOrK}

In this paper we have introduced novel notions of bisimulation for PAs. They are coarser than the existing bisimulations, and most importantly, we show that they agree with the logical equivalences induced by PCTL* and its sub logics. Even though we have not considered actions, it is worth noting that actions can be easily added, and all the (weak) bisimulations can be defined directly. On the other hand, the (weak) bisimulations are then strictly finer than the logical equivalences, because of the presence of these actions, similarly for simulations.

As future work, we plan to study decision algorithms for our new (strong and weak) bisimulation and simulation relations.

\section{ACKNOWLEDGEMENT}

The authors are supported by IDEA4CPS and the VKR Center of Excellence MT-LAB. The work has received support from the EU FP7-ICT projects TREsPASS (318003) and MEALS (295261), and the DFG Sonderforschungsbereich AVACS. Part of the work was done while the first author was with IT University of Copenhagen, Denmark. We thank Johann Schuster for detailed comments on an early version of this draft. 


\section{REFERENCES}

[1] A. Aziz, V. Singhal, and F. Balarin. It usually works: The temporal logic of stochastic systems. In $C A V$, pages 155-165, London, UK, 1995. Springer-Verlag.

[2] C. Baier, B. Engelen, and M. E. Majster-Cederbaum. Deciding bisimilarity and similarity for probabilistic processes. J. Comput. Syst. Sci., 60(1):187-231, 2000.

[3] C. Baier, H. Hermanns, J. Katoen, and V. Wolf. Comparative branching-time semantics for Markov chains. In CONCUR, pages 492-507. Springer, 2003.

[4] C. Baier and J.-P. Katoen. Principles of model checking. MIT Press, 2008.

[5] C. Baier, J.-P. Katoen, H. Hermanns, and V. Wolf. Comparative branching-time semantics for Markov chains. Inf. Comput., 200(2):149-214, 2005.

[6] A. Bianco and L. De Alfaro. Model checking of probabilistic and nondeterministic systems. In FSTTCS, pages 499-513. Springer, 1995.

[7] H. Boudali, P. Crouzen, and M. Stoelinga. A rigorous, compositional, and extensible framework for dynamic fault tree analysis. IEEE Transactions on Dependable and Secure Computing, 99(1), 2009.

[8] S. Cattani and R. Segala. Decision algorithms for probabilistic bisimulation. In CONCUR, pages 371$385,2002$.

[9] L. de Alfaro, R. Majumdar, V. Raman, and M. Stoelinga. Game relations and metrics. In LICS, pages 99-108, 2007.

[10] Y. Deng and R. Van Glabbeek. Characterising probabilistic processes logically. In LPAR, pages 278-293, Berlin, Heidelberg, 2010. Springer-Verlag.

[11] J. Desharnais, V. Gupta, R. Jagadeesan, and P. Panangaden. Weak bisimulation is sound and complete for pCTL ${ }^{*}$. Inf. Comput., 208(2):203-219, 2010.

[12] J. Desharnais, F. Laviolette, and M. Tracol. Approximate analysis of probabilistic processes: Logic, simulation and games. In QEST, pages 264-273, 2008.

[13] J. Desharnais, M. Tracol, and A. Zhioua. Computing distances between probabilistic automata. In QAPL, pages 148-162, 2011.

[14] P. Halmos. Measure theory, volume 1950. Springer-Verlag New York, 1974.

[15] H. Hansson and B. Jonsson. A calculus for communicating systems with time and probabilities. In IEEE RTSS, pages 278-287, 1990.

[16] H. Hansson and B. Jonsson. A logic for reasoning about time and reliability. Formal aspects of computing, 6(5):512-535, 1994.

[17] M. Hennessy. Exploring probabilistic bisimulations, part i. Formal Asp. Comput., 24(4-6):749-768, 2012.

[18] M. Hennessy and R. Milner. Algebraic laws for nondeterminism and concurrency. J. ACM, 32(1):137$161,1985$.

[19] M. R. Henzinger, T. A. Henzinger, and P. W. Kopke. Computing simulations on finite and infinite graphs. In FOCS, pages 453-462, Washington, DC, USA, 1995. IEEE Computer Society.

[20] H. Hermanns, A. Parma, R. Segala, B. Wachter, and L. Zhang. Probabilistic logical characterization. Inf. Comput., 209(2):154-172, 2011.

[21] B. Jonsson. Simulations between specifications of distributed systems. In CONCUR, pages 346-360, London, UK, 1991. Springer-Verlag.

[22] B. Jonsson and K. Larsen. Specification and refinement of probabilistic processes. In LICS, pages 266$277,1991$.

[23] B. Jonsson, K. Larsen, and Y. Wang. Probabilistic extensions of process algebras. In J. Bergstra, A. Ponse, and S. Smolka, editors, Handbook of Process Algebra, pages 685-710. Elsevier, 2001.

[24] J.-P. Katoen, T. Kemna, I. S. Zapreev, and D. N. Jansen. Bisimulation minimisation mostly speeds up probabilistic model checking. In TACAS, pages 87-101, 2007.

[25] K. Larsen and A. Skou. Bisimulation through probabilistic testing. Inf. Comput., 94(1):1-28, 1991.

[26] K. G. Larsen and A. Skou. Bisimulation through probabilistic testing. In POPL, pages 344-352, 1989.

[27] R. Milner. Communication and concurrency. Prentice Hall International Series in Computer Science, 1989.

[28] P. Panangaden. Labelled Markov Processes. Imperial College Press, 2009.

[29] A. Parma and R. Segala. Logical characterizations of bisimulations for discrete probabilistic systems. In FOSSACS, pages 287-301, Berlin, Heidelberg, 2007. Springer-Verlag. 
[30] A. Philippou, I. Lee, and O. Sokolsky. Weak bisimulation for probabilistic systems. In CONCUR, pages 334-349, 2000.

[31] W. Rudin. Real and complex analysis. Tata McGraw-Hill Education, 2006.

[32] J. Sack and L. Zhang. A general framework for probabilistic characterizing formulae. In $V M C A I$, pages 396-411, 2012.

[33] H. Schaefer, M. Wolff, and M. Wolff. Topological vector spaces, volume 3. Springer Verlag, 1999.

[34] R. Segala. Modeling and Verification of Randomized Distributed Realtime Systems. PhD thesis, MIT, 1995.

[35] R. Segala and N. A. Lynch. Probabilistic simulations for probabilistic processes. Nord. J. Comput., 2(2):250-273, 1995.

[36] R. Segala and A. Turrini. Comparative analysis of bisimulation relations on alternating and nonalternating probabilistic models. In QEST, pages 44-53, 2005.

[37] L. Song, L. Zhang, and J. Godskesen. Bisimulations meet pctl equivalences for probabilistic automata. In $C O N C U R$, pages 108-123, 2011.

[38] R. van Glabbeek and W. Weijland. Branching time and abstraction in bisimulation semantics. Journal of the ACM (JACM), 43(3):555-600, 1996.

[39] L. Zhang. Decision Algorithms for Probabilistic Simulations. PhD thesis, Universität des Saarlandes, 2008. 